\title{
Are maternal and child health initiatives helping to reduce under-five mortality in Ghana? Results of a quasi-experimental study using coarsened exact matching
}

\author{
Augusta Kolekang ${ }^{1,2^{*}}$, Bismark Sarfo ${ }^{2}$, Anthony Danso-Appiah ${ }^{2}$, Duah Dwomoh ${ }^{2}$ and Patricia Akweongo ${ }^{2}$
}

\begin{abstract}
Background: Despite a $53 \%$ decline in under-five mortality (U5M) worldwide during the period of the Millennium Development Goals (MDGs), U5M remains a challenge. Under-five mortality decline in Ghana is slow and not parallel with the level of coverage of child health interventions. The interventions promoted to improve child survival include early initiation of breastfeeding, clean postnatal care, and skilled delivery. This study sought to assess the effectiveness of these interventions on U5M in Ghana.
\end{abstract}

Methods: A quasi-experimental study was conducted using secondary data of the 2008 and 2014 Ghana Demographic and Health Surveys. Coarsened Exact Matching and logistic regression were done. The interventions assessed were iron intake, early initiation of breastfeeding, clean postnatal care, hygienic disposal of stool, antenatal care visits, skilled delivery, intermittent preventive treatment of malaria in pregnancy, and tetanus toxoid vaccine.

Results: There were 2,045 children under-five years and 40 (1.9\%) deaths in 2008. In 2014, the total number of children under-five years was 4,053, while deaths were 53(1.2\%). In 2014, children less than one month old formed $1.6 \%$ of all children under-five years, but $47.8 \%$ of those who died. Mothers who attended four or more antenatal care visits were $78.2 \%$ in 2008 and $87.0 \%$ in 2014. Coverage levels of improved sanitation and water connection in the home were among the lowest, with $11.6 \%$ for improved sanitation and $7.3 \%$ for water connection in the home in 2014. Fifty-eight (58), 1.4\%, of children received all the eight (8) interventions in 2014, and none of those who received all these interventions died. After controlling for potential confounders, clean postnatal care was associated with a $66 \%$ reduction in the average odds of death ( $\mathrm{aOR}=0.34,95 \% \mathrm{Cl}: 0.14-0.82)$, while early initiation of breastfeeding was associated with a $62 \%$ reduction in the average odds of death ( $a \mathrm{OR}=0.38,95 \% \mathrm{Cl}: 0.21-0.69)$.

Conclusions: Two (clean postnatal care and early initiation of breastfeeding) out of eight interventions were associated with a reduction in the average odds of death. Thus, a further decline in under-five mortality in Ghana will require an increase in the coverage levels of these two high-impact interventions.

Keywords: Child health, Under-five, Mortality, Interventions, Matching, And effectiveness

*Correspondence: augustakolekang@yahoo.com

${ }^{1}$ University for Development Studies, Accra, Tamale, Ghana

Full list of author information is available at the end of the article

(c) The Author(s) 2021. Open Access This article is licensed under a Creative Commons Attribution 4.0 International License, which permits use, sharing, adaptation, distribution and reproduction in any medium or format, as long as you give appropriate credit to the original author(s) and the source, provide a link to the Creative Commons licence, and indicate if changes were made. The images or other third party material in this article are included in the article's Creative Commons licence, unless indicated otherwise in a credit line to the material. If material is not included in the article's Creative Commons licence and your intended use is not permitted by statutory regulation or exceeds the permitted use, you will need to obtain permission directly from the copyright holder. To view a copy of this licence, visit http://creativecommons.org/licenses/by/4.0/. The Creative Commons Public Domain Dedication waiver (http://creativeco $\mathrm{mmons}$.org/publicdomain/zero/1.0/) applies to the data made available in this article, unless otherwise stated in a credit line to the data. 


\section{Research in context} What is already known?

- Increasing usage of child health interventions has been advocated to achieve a rapid decline in mortality. However, the effectiveness of these interventions can be sub-optimal, and some interventions are associated with an increased risk of death of children.

\section{What are the new findings?}

- A quasi-experimental study design with coarsened exact matching was used to assess multiple interventions using secondary data.

\section{What do the new findings imply?}

- Only a few interventions were associated with a reduction in mortality.

- To achieve a further decline in mortality, coverage of early initiation of breastfeeding and clean postnatal care should be increased since their coverage levels are low.

- Attention should be paid to children at higher risk of dying, including those with preceding birth interval less than two years, neonates, multiple births, and children from polygamous homes.

\section{Introduction}

Under-five mortality (U5M) remains a major public health problem despite a $53 \%$ decline globally between 1990 and 2015 [1]. Decline of U5M in Ghana is considered slow [2-4]. Thus, it could not achieve the Millennium Development Goal (MDG) 4 target $[4,5]$ despite several efforts at achieving it. According to Schieber, Cashin [2], Ghana's under-five mortality rate is not commensurate with its high health care spending compared to other countries at the global level [2].

Variations in socio-demographic factors, including household, maternal, and child factors $[6,7]$, the prevalence of diseases and risk factors, and the impact of interventions [8-10] underpin the variations in mortality decline. To this end, maternal, neonatal, child health, and nutrition interventions have been advocated to reduce U5M. In Ghana, child health interventions include antenatal care visits, clean postnatal care, early initiation of breastfeeding, health facility delivery, skilled delivery, and household ownership of mosquito net. In addition, several strategies, including the Community-based Health Planning Services (CHPS) and the National Health Insurance Scheme (NHIS), have been implemented to increase access to and coverage of child health interventions and to improve survival [3]. These strategies resulted in increased coverage levels of most vaccines to $80 \%$ in 2014 [11]. However, coverage levels of some other interventions, including clean postnatal care, improved sanitation, and water connection in the home, were below $30 \%$ in Ghana in 2014 [3].

While low coverage of interventions leads to low impact [12-14], the impact of interventions can be low even when coverage is high if the quality of the interventions is poor or there are adverse events associated with the interventions. Poorer child survival has been documented with health facility and skilled delivery compared to home deliveries [15], iron intake [16], diphtheria, pertussis, and tetanus (DPT) vaccine $[17,18]$, and malaria vaccine $[19,20]$. To achieve rapid mortality decline and possibly achieve the Sustainable Development Goals (SDG) 3 target 2, information on interventions with the potential to rapidly reduce mortality is required. This study, therefore, sought to assess the effectiveness of the child health interventions implemented in Ghana. The null hypothesis was that no intervention was associated with a decrease in U5M in Ghana during the period of the evaluation.

\section{Methods \\ Study design}

This was a quasi-experimental study design used to assess the effectiveness of eight [8] interventions. The Mosley and Chen framework for studying child survival in developing countries was adapted for this study [21]. The interventions were antenatal care visits, neonatal tetanus toxoid vaccine, clean postnatal care, hygienic disposal of stool, early initiation of breastfeeding, intermittent preventive treatment of malaria in pregnancy (IPT-p), iron intake, and skilled delivery. These are interventions expected to be implemented before, during, or shortly after birth, and that are therefore likely to have preceded (temporal association between exposure and outcome) death for those children who received them [22]. In addition, an overall or composite intervention group was created using children who received all eight [8] interventions. Definition of interventions can be found in the 2014 Ghana Demographic and Health Survey report [11]. For each child that received an intervention (treated), a control child who did not receive the intervention (untreated) was matched using coarsened exact matching [23-26]. 


\section{Study area}

The study area was Ghana, a country in sub-Saharan Africa (SSA). There were ten [10] political regions and 216 districts in Ghana in 2008 and 2014. At the last census in 2010, the total population of Ghana was about 25 million [27]. The agriculture and services sectors contributed the most to the economy during the period of the evaluation [28]. On general health, the burden of illness or injury was $14 \%$ in 2014, with individuals 50 years old and above $(22.4 \%)$ and children $0-5$ years $(20.3 \%)$ reporting the highest burden [28].

Regarding the eating habits of Ghanaians, results from the literature are inconsistent. While Buxton [29] recorded that $63 \%$ of Junior High Students in Ghana reported skipping breakfast on school-going days, Intiful and Lartey [30] recorded $86 \%$ of students aged 6-19 years old in the Eastern Region of Ghana taking breakfast on the day they were interviewed. $45 \%$ of adolescents reported eating two home-cooked meals a day, and $34 \%$ preferred a soft drink during the day [29]. Rural dwellers consumed more starchy foods, while urban dwellers consumed more animal-based foods [31]. Skipping breakfast, low consumption of fruits and vegetables, and high consumption of energy-dense foods have also been reported among university students in Ghana [32].

\section{Study participants}

The study population was children under-five years old in the Ghana Demographic and Health Surveys (GDHS) data sets born between 2003 and 2014. The analyses were restricted to usual household members and last births. The last birth restriction was done because interventions including antenatal care visits, iron intake, and clean postnatal care were measured for only the last births. It would also reduce the effect of recall bias on the study results. Only usual household members were also used because some interventions were not measured for nonusual household members.

\section{Data and source of data}

Secondary data from the 2008 and 2014 GDHS were used. The data were collected by the Ghana Statistical Service (GSS). The GDHSs are complex household surveys, and the data were collected using a cross-sectional descriptive study design. Individuals were selected using a multistage cluster sampling technique [3]. The data are nationally representative, and response rates were over $90 \%$ for both surveys used in this analysis [3]. Data sets of the 2008 and 2014 surveys were pooled for regression analysis. The years 2008 and 2014 were chosen because they have the data sets closest to the transition from the MDGs to the SDGs. Information from the study will therefore provide an understanding of the interventions

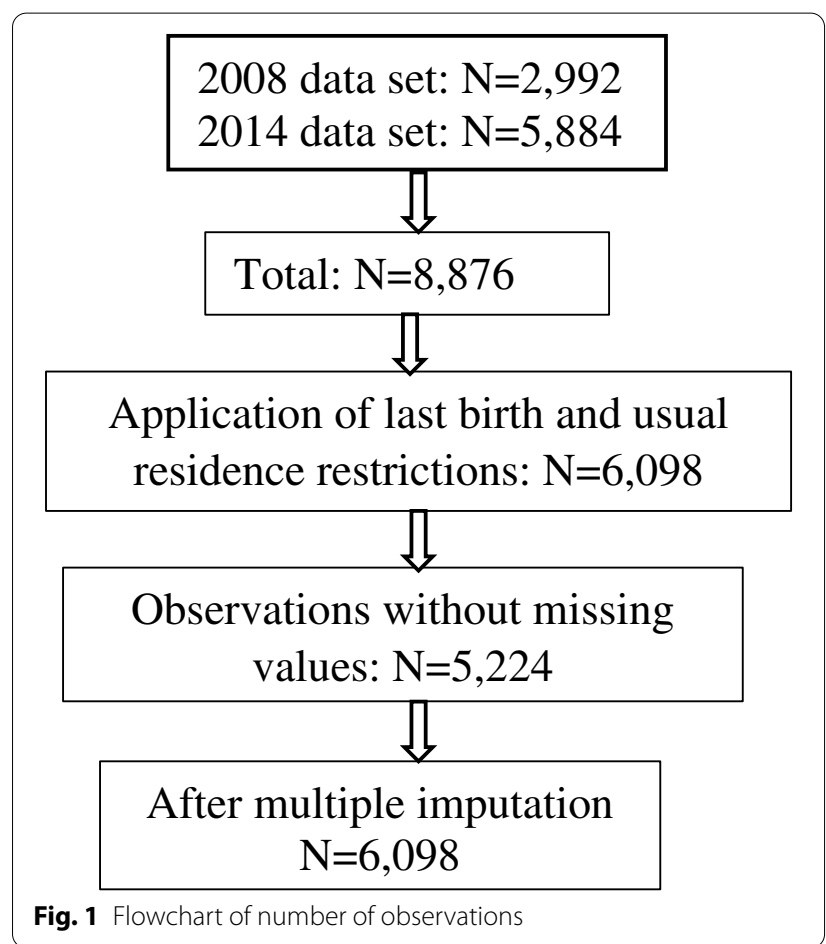

that contributed to mortality reduction towards the end of the MDGs and, thus, should be focused on during the SDGs period to achieve rapid mortality reduction.

The Household Recode (HR) and Kids Recode (KR) files were used for this analysis. The KR file was the main data set, and it contained information about children under-five years old at the start of the data collection whose mothers were interviewed [3]. The 2008 GDHS data set had 2,882 observations and 1,010 variables, while the 2014 had 5,884 observations and 1,159 variables (Fig. 1). The Stata versions of the data sets were downloaded from the DHS Program's website (https://dhspr ogram.com). The HR data sets were merged with the KR data sets so that household insecticide-treated nets/ indoor residual spraying (ITN/IRS) information from the HR data set could be added to the KR data set. Household insecticide-treated nets/indoor residual spraying is an intervention and needed to be adjusted for in the analysis. The data sets of 2008 and 2014 were combined to obtain the final data set for the analysis.

$48 \%$ (48\%) of observations had at least one missing value for the covariates to be adjusted for, and therefore, multiple imputation was done before the regression analyses were done. Imputation was done to preserve the sample size and representativeness of the data. Variables with missing data were birth interval (16 observations), number of co-wives (832 observations), mode of delivery of child ( 2 observations), child's weight (19 observations), employment status of mother (13 observations), NHIS 
status (1 observation), maternal BMI $(2,601)$, anaemia (204), height $(2,008)$ and child given other milk within three days after birth (58). The information on the missing data is presented in Additional file 3. Twenty (20) imputations were done using chain imputation and logistic regression. The imputation data format was marginal long, and the seed set was 200. Variables used in the multiple imputation were all variables without missing data that were used in the analysis. The design feature, weighting, was factored into the multiple imputation model. The Stata syntax of the multiple imputation is presented in Additional file 1.

\section{Study variables}

The variables used in this analysis were socio-economic and demographic factors and interventions. Socio-economic factors were maternal and household variables, including maternal education, marital status, religion, ethnicity, household wealth, household size, number of children under-five years in a household, region, NHIS status, and place of residence. Demographic factors comprised child and maternal variables, including maternal age, child's age and birth weight, birth interval, birth order, and multiple birth. Interventions were antenatal visit, IPT-p, iron intake, tetanus toxoid vaccine, skilled delivery, clean postnatal care, early initiation of breastfeeding, hygienic disposal of stool, water connection in the home, improved water source, improved sanitation, and ITN/IRS. The year of the survey was also included. The definition of variables is based on the 2014 GDHS report [3].

\section{Definition of interventions}

Intermittent preventive treatment of malaria in pregnancy (IPT-p): Percent of pregnant women receiving $2+$ doses of sulphadoxine-pyrimethamine/fansidar during pregnancy.

Iron intake: Percent of pregnant women taking an iron supplement daily for at least 90 days.

Tetanus toxoid vaccination: Percent of women who received two injections during the pregnancy of her last birth, or two or more injections (the last within 3 years of the last live birth), or three or more injections (the last within 5 years of the last birth), or four or more injections (the last within 10 years of the last live birth), or five or more injections at any time prior to the last birth.

Skilled delivery: Percent of children born with a skilled attendant present, including a medical doctor, nurse/ midwife, and community health officer/nurse.

Clean postnatal care: Percent of neonates who received a preventive postnatal visit within $48 \mathrm{~h}$ of birth.

Early initiation of breastfeeding: Percent of children who started breastfeeding within $1 \mathrm{~h}$ of birth.
Hygienic disposal of stool: Percent of children's stools that are disposed of safely. Stools are considered to be disposed of safely if: (1) the child always uses a toilet/ latrine, (2) the feaces are thrown in the toilet/latrine, or (3) the feaces are buried in the yard.

Antenatal care visit: Percentage of women with a birth in the last 5 years receiving antenatal care from a skilled provider for the most recent birth.

\section{Pre-processing of data}

Coarsened Exact Matching [23] was used to pre-process (match) the data to ensure balance in pre-treatment variables (covariates) between the treated and untreated groups. Covariate balance was assessed using linear 1 (L1) statistic. Linear $1=0$ means perfect covariate balance between treated and untreated groups, while $\mathrm{L} 1=1$ means complete incompatible treatment and comparison groups with respect to the covariates. The closer the values of L1 to zero, the better the covariate balance [23]. Information on the sample size and covariate balance before and after matching are included in Additional file 2.

CEM puts observations into groups based on factors that influenced the decision to receive treatment. The groups were chosen by the first author to reflect clinically important categories and to be consistent with that in the GDHS reports. Observations in similar groups were matched, and treated and untreated individuals who did not have matches were deleted from the data set and the data analyzed as unmatched data. The covariates used in the matching process were variables that predicted intervention use from the literature review and were also associated with each intervention from the chi-square test done in this data analysis (Additional file 2).

\section{Analysis}

Descriptive statistics were done and presented as numbers (percentages) by survival status (died or lived), and chi-squared test was used to assess the differences each for the years 2008 and 2014. Logistic regression was also fitted to assess factors associated with U5M after the multiple imputation using combined data of 2008 and 2014. The design features (stratification, clustering and weighting, and selection of primary sampling units) of the GDHS were factored into all the analyses. Also, the difference in women's population between the two time periods was accounted for in the analysis. De-normalized weights were used to account for the differences in the population of women in the reproductive age in the population at the time of each survey. The de-normalized weight was calculated as $=\psi \mathrm{X} \phi 15-49 / \phi^{\mathrm{S}} 15-49$ [33]. Where $\psi$ is the DHS sampling weight for women. $\phi 15-49$ is the population of women in the country at the 
time of each survey, while $\phi^{\mathrm{S}} 15-49$ is the total number of women 15-49 years interviewed during the data collection for each of the surveys as reported in the GDHS reports [11,34].

Potential confounders adjusted for were those related to mortality and/or exposure from the conceptual framework/directed acyclic graphs (DAGs) (Additional file 4) and are not intermediates in the causal pathway of the exposure and outcome $[35,36]$. Robust estimates of odds ratios (ORs) with 95\% confidence intervals were reported.

For the analysis of the effect of interventions on U5M, under the null hypothesis that no intervention had an effect on U5M, logistic regression was fitted using the pre-processed CEM data. Each intervention (antenatal care visit, IPT-p, iron intake, tetanus toxoid vaccine, skilled delivery, clean postnatal care, early initiation of breastfeeding, and hygienic disposal of stool) was the exposure, accounting for design features and controlling for potential confounders. Potential confounders adjusted for were those identified from the DAGs. Average treatment effects were reported as OR with $95 \%$ CI and at a 2-tailed $\alpha$ level of 0.05 [37]. Analyses were performed using Stata version 13 [37]. The CEM syntax and algorithm are included in Additional file 1.

\section{Sensitivity analysis \\ Complete case analysis without maternal nutrition factors}

When logistic regression was fitted with the completed cases, the total number of observations was 5,224, and early initiation of breastfeeding, child's age, multiple birth, birth interval, number of children under-five years old in the household, and polygamy (co-wives) were associated with U5M from the adjusted analysis.

\section{Multiple imputation with maternal nutrition factors}

After multiple imputation, when maternal anaemia and Body Mass Index (BMI) were added, the sample size was reduced from 6,098 to 3,165 observations with very wide $95 \%$ confidence intervals. The variable, water connection in the home, was omitted from the regression model. Body Mass Index and anaemia were not statistically significant, while household size, child's age, multiple birth, maternal education, household indoor residual spraying and bed net ownership, and early initiation of breastfeeding were statistically significant. National Health Insurance Scheme status had borderline significance, $\mathrm{p}=0.05$. The confidence interval for multiple births ranged from 9.83 to 417.8 .

\section{Coarsened exact matching with completed cases without maternal nutrition factors}

For the CEM analysis with completed cases, the sample size after matching for the regression analysis ranged from 4,914 to 6,043 observations. Only early initiation of breastfeeding was associated with U5M reduction from both the crude and adjusted analysis. Clean postnatal care and hygienic disposal of stool were significant from the crude analysis but not from the adjusted analysis.

\section{Results}

\section{Descriptive statistics}

There were 2,045 children under-five years and 40 (1.9\%) deaths in 2008. In 2014, the total number of children was 4,053 , and deaths were 53 constituting $1.2 \%$ (Table 1 ). In 2008 , children less than one month constituted $2.2 \%$ of all children under-five years, but $46.0 \%$ among those who died. In 2014, children less than one month old formed $1.6 \%$ of all children, but $47.8 \%$ of those who died. About $78.2 \%$ of mothers attended four or more antenatal care visits in 2008, increasing to $87.0 \%$ in 2014 (Table 2). The interventions with the lowest coverage levels were improved sanitation and water connection in the home. The coverage level of improved sanitation was $7.8 \%$ in 2008 and $11.6 \%$ in 2014, while water connection in the home was $9.7 \%$ in 2008 and $7.3 \%$ in 2014. About $58(1.4 \%)$ of children received all eight (8) interventions in 2014, and none of those who received all the eight interventions died.

\section{Interventions and under-five mortality}

The crude and adjusted ORs of the effect of the interventions are presented in Tables 3 and 4. From the crude analysis, average odds of death was reduced by $68 \%$ among those with early initiation of breastfeeding (OR $=0.32,95 \% \mathrm{CI}: 0.19-0.54)$ and also $68 \%$ among children under-five years who had clean postnatal care within 2 days $(\mathrm{OR}=0.32,95 \% \mathrm{CI}$ : 0.16 0.65 ). After adjusting for potential confounders, early initiation of breastfeeding reduced odds of death by $62 \%(\mathrm{aOR}=0.38,95 \% \mathrm{CI}: 0.21-0.69)$, while clean postnatal care was associated with a $66 \%$ reduction in the average odds of death (aOR $=0.34,95 \% \mathrm{CI}$ : $0.14-$ 0.82 ) (Tables 3 and 4). Skilled delivery was not associated with $\mathrm{U} 5 \mathrm{M}$ from the crude $(\mathrm{OR}=1.10,95 \% \mathrm{CI}$ : $0.55-2.20)$ and adjusted analysis $(\mathrm{aOR}=1.77,95 \% \mathrm{CI}$ : $0.60-5.25)$. Antenatal care visits was associated with U5M in the crude analysis (OR $=2.52$, $95 \%$ CI: $1.18-$ 5.36) but not in the adjusted analysis $(\mathrm{aOR}=1.59$, 95 \%CI: 0.71-3.57).

\section{Socio-demographic factors and interventions associated with under-five mortality}

Results of the association of child, maternal and sociodemographic factors with U5M are presented in Table 5. The crude and adjusted ORs and $95 \%$ CI for the associations are presented. On child factors, after controlling 
Table 1 Background factors and mortality among children under-five years old in 2008 and $2014(n=6,098)$

\begin{tabular}{|c|c|c|c|c|c|c|c|c|}
\hline \multirow{3}{*}{$\begin{array}{l}\text { Year } \\
\text { Characteristics }\end{array}$} & \multicolumn{4}{|c|}{ Baseline (2008) } & \multicolumn{4}{|c|}{ End line (2014) } \\
\hline & Total & Alive (98.1 \%) & Died (1.9\%) & P-value & Total & $\begin{array}{l}\text { Alive } \\
\text { (98.8\%) }\end{array}$ & $\operatorname{Died}(1.2 \%)$ & P-value \\
\hline & $n(\%)$ & $n(\%)$ & $n(\%)$ & & $\mathrm{n}(\%)$ & $n(\%)$ & $\mathrm{n}(\%)$ & \\
\hline \multicolumn{9}{|l|}{ Age(months) } \\
\hline$<1$ & $46(2.2)$ & $26(1.4)$ & $20(46.0)$ & $<0.001$ & $68(1.6)$ & $41(1.0)$ & $27(47.8)$ & $<0.001$ \\
\hline 1 to 5 & $294(14.1)$ & $288(14.1)$ & $6(13.5)$ & & $545(12.7)$ & $538(12.7)$ & $7(12.2)$ & \\
\hline 6 to 11 & $319(15.2)$ & $312(15.1)$ & $7(16.9)$ & & $577(14.8)$ & $569(14.7)$ & $8(15.2)$ & \\
\hline 12 to 59 & $1386(68.6)$ & $1379(69.4)$ & $7(23.6)$ & & $2863(71.0)$ & 2852(71.5) & $11(24.7)$ & \\
\hline \multicolumn{9}{|l|}{ Sex } \\
\hline Male & $1043(51.5)$ & 1016(51.2) & $27(69.7)$ & 0.03 & $2118(52.7)$ & 2095(52.8) & $23(43.1)$ & 0.22 \\
\hline Female & $1002(48.4)$ & $989(48.8)$ & $13(30.3)$ & & $1935(47.3)$ & 1905(47.2) & $30(56.9)$ & \\
\hline \multicolumn{9}{|l|}{ Birth size) } \\
\hline$>=2.5 \mathrm{~kg}$ & 1763(88.0) & 1730(88.0) & 33(86.8) & 0.83 & $3545(88.5)$ & $3503(88.5)$ & $42(84.9)$ & 0.45 \\
\hline$<2.5 \mathrm{~kg}$ & $266(12.0)$ & $260(12.0)$ & $6(13.2)$ & & $505(11.5)$ & $495(11.5)$ & $10(15.1)$ & \\
\hline \multicolumn{9}{|c|}{ Preceding birth interval } \\
\hline$>=2$ years & $1852(90.5)$ & 1818(90.7) & $34(81.5)$ & 0.09 & 3700(90.8) & $3654(90.9)$ & $46(86.1)$ & 0.37 \\
\hline$<2$ years & $188(9.5)$ & $182(9.3)$ & $6(18.5)$ & & $342(9.2)$ & $335(9.2)$ & $7(13.9)$ & \\
\hline \multicolumn{9}{|l|}{ Birth order } \\
\hline$<3$ & $1191(59.7)$ & 1177(60.0) & $14(42.6)$ & 0.04 & 2318 (59.8) & 2295(59.9) & $23(44.6)$ & 0.06 \\
\hline$(>=3)$ & 854 (40.3) & $828(40.0)$ & $26(57.4)$ & & 1735 (40.3) & $1705(40.1)$ & $30(55.4)$ & \\
\hline \multicolumn{9}{|l|}{ Gestation } \\
\hline Singleton & 1994 (97.5) & 1959(97.7) & $35(85.7)$ & $<0.001$ & 3951 (97.5) & $3902(97.5)$ & $49(93.3)$ & 0.05 \\
\hline Multiple birth & $51(2.5)$ & $46(2.3)$ & $5(14.3)$ & & $102(2.5)$ & $98(2.5)$ & $4(6.7)$ & \\
\hline \multicolumn{9}{|l|}{ Delivery type } \\
\hline Vaginal & 1913(92.9) & 1877(93.1) & $36(86.1)$ & NA & $3611(86.8)$ & 48(89.6) & 3563(86.8) & 0.65 \\
\hline Caesarean & $130(7.1)$ & $126(7.0)$ & $4(13.9)$ & & $442(10.5)$ & $437(13.2)$ & $5(10.5)$ & \\
\hline \multicolumn{9}{|l|}{ Had NHIS } \\
\hline No & 1198(60) & 1174(59.9) & $24(64.9)$ & 0.56 & 1238(33.0) & 1219(32.9) & 19(39.4) & 0.38 \\
\hline Yes & $847(40)$ & $831(40.1)$ & $16(25.1)$ & & $2814(67.0)$ & $2780(67.1)$ & $34(60.6)$ & \\
\hline \multicolumn{9}{|l|}{ Household size } \\
\hline Less than 6 & 1099 (57.4) & 1082(57.6) & $17(45.0)$ & 0.14 & 2255 (59.1) & $2230(59.3)$ & $25(46.1)$ & 0.1 \\
\hline$>=6$ & $946(42.6)$ & $923(42.4)$ & $23(55.0)$ & & $1798(40.9)$ & $1770(40.7)$ & $28(53.9)$ & \\
\hline \multicolumn{9}{|l|}{ Region } \\
\hline Northern belt & $676(22.7)$ & $657(22.6)$ & $19(33.8)$ & 0.20 & $1363(19.0)$ & $1341(18.9)$ & $22(26.1)$ & 0.21 \\
\hline Middle belt & $669(37.6)$ & $657(37.6)$ & $12(38.8)$ & & $1221(36.0)$ & $1205(35.9)$ & $16(42.2)$ & \\
\hline Southern belt & $700(39.6)$ & $691(39.8)$ & $9(27.5)$ & & $1469(45.0)$ & $1454(45.2)$ & $15(31.7)$ & \\
\hline \multicolumn{9}{|c|}{ Rural/urban residence } \\
\hline Urban & $725(40.3)$ & $712(40.4)$ & 13(35.2) & 0.54 & 1669(46.2) & $1651(46.3)$ & 18(39.0) & 0.38 \\
\hline Rural & $1320(59.7)$ & 1293(59.6) & $27(64.8)$ & & $2384(53.8)$ & $2349(53.7)$ & $35(61.0)$ & \\
\hline \multicolumn{9}{|l|}{ Wealth quintile } \\
\hline Poorest & $617(23.2)$ & $599(23.0)$ & $18(33.5)$ & 0.67 & $1279(21.7)$ & $1258(21.5)$ & $21(33.0)$ & 0.42 \\
\hline Poorer & $450(22.0)$ & $443(22.1)$ & $7(19.6)$ & & $865(20.1)$ & $851(20.1)$ & $14(24.0)$ & \\
\hline Middle & $348(18.6)$ & $342(18.7)$ & $6(18.6)$ & & $750(19.6)$ & $742(19.6)$ & $8(16.2)$ & \\
\hline Richer & $368(20.8)$ & $363(20.8)$ & $5(18.7)$ & & $641(19.6)$ & 635(19.6) & $6(16.4)$ & \\
\hline Richest & $262(15.4)$ & $258(15.5)$ & $4(9.6)$ & & $518(19.1)$ & $514(19.2)$ & $4(10.5)$ & \\
\hline \multicolumn{9}{|l|}{ Mother's age } \\
\hline$<35$ & $1623(80.7)$ & 1597(80.9) & $26(72.0)$ & 0.18 & $3148(78.0)$ & $3110(78.1)$ & $38(71.5)$ & 0.35 \\
\hline 35 to 49 & $422(19.3)$ & 408(19.1) & $14(28.0)$ & & $905(22.1)$ & $890(21.9)$ & $15(28.5)$ & \\
\hline Maternal educatio & & & & & & & & \\
\hline
\end{tabular}


Table 1 (continued)

\begin{tabular}{|c|c|c|c|c|c|c|c|c|}
\hline \multirow{3}{*}{$\begin{array}{l}\text { Year } \\
\text { Characteristics }\end{array}$} & \multicolumn{4}{|c|}{ Baseline (2008) } & \multicolumn{4}{|c|}{ End line (2014) } \\
\hline & Total & Alive (98.1 \%) & Died (1.9\%) & P-value & Total & $\begin{array}{l}\text { Alive } \\
\text { (98.8\%) }\end{array}$ & $\operatorname{Died}(1.2 \%)$ & P-value \\
\hline & $\mathrm{n}(\%)$ & $n(\%)$ & $n(\%)$ & & $\mathrm{n}(\%)$ & $n(\%)$ & $n(\%)$ & \\
\hline None & $748(31.2)$ & $730(31.1)$ & $18(37.1)$ & 0.0001 & $1371(26.6)$ & $1347(26.5)$ & $24(36.7)$ & 0.14 \\
\hline Primary & $481(24.2)$ & $464(23.6)$ & $17(50.6)$ & & $824(19.5)$ & 814(19.4) & $10(25.9)$ & \\
\hline Secondary or higher & $816(44.6)$ & $811(45.3)$ & $5(12.3)$ & & $1858(53.9)$ & $1839(54.1)$ & $19(37.4)$ & \\
\hline \multicolumn{9}{|l|}{ Religion } \\
\hline Orthodox & $530(25.2)$ & $522(25.3)$ & $8(19.7)$ & 0.04 & $960(21.7)$ & $948(21.7)$ & $12(23.3)$ & 0.54 \\
\hline Pentecostal & $851(46.2)$ & $838(46.4)$ & $13(36.7)$ & & $1937(54.5)$ & $1917(54.7)$ & $20(44.3)$ & \\
\hline Islam & $399(18.2)$ & $390(18.2)$ & $9(18.7)$ & & $836(16.8)$ & $818(16.7)$ & $18(24.7)$ & \\
\hline Others & $265(10.4)$ & $255(10.1)$ & $10(24.9)$ & & $320(7.0)$ & $317(7.0)$ & $3(7.7)$ & \\
\hline \multicolumn{9}{|l|}{ Ethnicity } \\
\hline Akan & $793(46.5)$ & $779(46.5)$ & $14(45.3)$ & 0.98 & $1515(46.5)$ & $1502(46.7)$ & $13(33.7)$ & 0.27 \\
\hline Mole-Dagbani & $528(20.4)$ & $515(20.4)$ & $13(20.2)$ & & $1090(17.3)$ & 1074(17.3) & $16(20.9)$ & \\
\hline Others(Ga/Dangbe/Eve) & $724(33.1)$ & $711(33.1)$ & $13(34.5)$ & & $1448(36.2)$ & 1424(36.0) & $24(45.4)$ & \\
\hline \multicolumn{9}{|l|}{ Married } \\
\hline No & $575(31.3)$ & $566(31.4)$ & $9(28.3)$ & & $1370(37.3)$ & $1349(37.3)$ & 21(44.6) & 0.36 \\
\hline Yes & $1470(68.7)$ & $1439(68.7)$ & $31(71.7)$ & 0.72 & $2683(62.7)$ & $2651(62.7)$ & $32(55.5)$ & \\
\hline \multicolumn{9}{|l|}{ Polygamous home } \\
\hline No & $1428(80.5)$ & 1406(80.8) & $22(68.2)$ & & $2801(84.8)$ & $2773(84.9)$ & $28(75.5)$ & 0.1 \\
\hline Yes & $386(19.5)$ & $374(19.2)$ & $12(31.8)$ & 0.09 & $651(15.2)$ & $639(15.1)$ & $12(24.5)$ & \\
\hline \multicolumn{9}{|l|}{ Mother's employment status } \\
\hline Not employed & $248(12.5)$ & $241(12.3)$ & $7(19.5)$ & 0.20 & $811(19.9)$ & $800(19.9)$ & $11(22.2)$ & 0.72 \\
\hline Employed & $1786(87.5)$ & 1753(87.7) & $33(80.5)$ & & $3240(80.1)$ & $3198(80.1)$ & $42(77.8)$ & \\
\hline \multicolumn{9}{|c|}{ Number of children $<5$ years in household } \\
\hline $1-2$ & $1769(86.9)$ & 1736(87.0) & $33(82.3)$ & 0.45 & $3557(88.7)$ & $3509(88.6)$ & $48(92.2)$ & 0.39 \\
\hline $3-9$ & $276(13.1)$ & 269(13.0) & $7(17.7)$ & & $496(11.3)$ & $491(11.4)$ & $5(7.8)$ & \\
\hline \multicolumn{9}{|l|}{ Maternal anaemia } \\
\hline Not anaemic & $788(38.9)$ & $776(39.0)$ & $12(34.2)$ & 0.58 & 1169(56.7) & 1153(56.6) & $16(64.8)$ & 0.45 \\
\hline Anaemic & 1204(61.1) & $1177(61.0)$ & $27(65.8)$ & & $876(43.4)$ & $867(43.4)$ & $9(35.2)$ & \\
\hline \multicolumn{9}{|c|}{ Maternal Body Mass Index (BMI) } \\
\hline Normal & $1120(63.2)$ & $1110(63.3)$ & $10(59.2)$ & 0.77 & 1034(54.9) & $1031(54.9)$ & $3(35.9)$ & 0.03 \\
\hline Abnormal & $609(36.8)$ & $603(36.8)$ & $6(40.8)$ & & $734(45.2)$ & $730(45.1)$ & $4(64.2)$ & \\
\hline \multicolumn{9}{|l|}{ Maternal height } \\
\hline$>145 \mathrm{~cm}$ & 1989(98.6) & 1950(98.7) & 39(96.9) & NA & 2052(99.0) & 2028(99.0) & 24(97.4) & NA \\
\hline$<=145 \mathrm{~cm}$ & 29(1.4) & $28(1.3)$ & $1(3.1)$ & & $20(1.0)$ & 19(1.0) & $1(2.6)$ & \\
\hline \multicolumn{9}{|c|}{ Infant given other milk with 3 days after birth } \\
\hline No & 1961(97.0) & 1929(96.9) & $32(100)$ & NA & 3974(98.9) & 3940(98.8) & $34(100)$ & 0.61 \\
\hline Yes & $56(3.0)$ & $56(3.0)$ & $0(0.0)$ & & $49(1.2)$ & $49(1.2)$ & $0(0.0)$ & \\
\hline Total & $2045(100)$ & 2005(100) & $40(100)$ & & $4,053(100)$ & $4000(100)$ & $53(100)$ & \\
\hline
\end{tabular}

Birth interval, child's weight, NHIS coverage, mother's employment status, polygamous home, anaemia, maternal height, BMI, and child given other milk within three days after birth had missing data. Details of the missing data for 2008 and 2014 are presented in supplementary file 2

Source: 2008 and 2014 Ghana Demographic and Health Surveys

for potential confounders, compared to children less than 1month old, odds of death was reduced by $98 \%$ $(\mathrm{aOR}=0.02,95 \% \mathrm{CI}: 0.01-0.04)$ among children 1-5 months. Also, a $98 \%$ reduction of odds of death was reported among those 6-11 months $(\mathrm{aOR}=0.02,95 \%$
CI: $0.01-0.05)$ and $99 \%$ among those 12-59 months $(\mathrm{aOR}=0.01,95 \% \mathrm{CI}: 0.002-0.01)$ relative to those less than 1 month old. Children who were multiple births were 7 times more likely to die compared to singleton births $(\mathrm{aOR}=6.55,95 \% \mathrm{CI}: 1.56-27.57)$, while those 
Table 2 Coverage of interventions and mortality among children under-five years old $(n=6,098)$

\begin{tabular}{|c|c|c|c|c|c|c|c|c|}
\hline \multirow{2}{*}{$\begin{array}{l}\text { Year } \\
\text { Intervention }\end{array}$} & \multicolumn{4}{|c|}{ Baseline (2008) } & \multicolumn{4}{|c|}{ End line (2014) } \\
\hline & $\begin{array}{l}\text { Total } \\
\text { n (\%) }\end{array}$ & $\begin{array}{l}\text { Alive } \\
\text { n(\%) }\end{array}$ & $\begin{array}{l}\text { Died } \\
\text { n (\%) }\end{array}$ & P-value & $\begin{array}{l}\text { Total } \\
\text { n (\%) }\end{array}$ & $\begin{array}{l}\text { Alive } \\
\mathrm{n}(\%)\end{array}$ & $\begin{array}{l}\text { Died } \\
\text { n (\%) }\end{array}$ & P-value \\
\hline Water $<30$ min & $1600(77.5)$ & $1566(77.5)$ & $34(80.0)$ & 0.72 & $2813(64.2)$ & $2772(64.1)$ & $41(72.7)$ & 0.30 \\
\hline Water connected & $175(9.7)$ & 173(9.9) & $2(17.0)$ & NA & $294(7.3)$ & $288(7.2)$ & $6(11.8)$ & 0.30 \\
\hline Improved sanitation & $156(7.8)$ & $152(7.8)$ & $4(8.7)$ & NA & $404(11.6)$ & $400(11.7)$ & $4(5.3)$ & 0.10 \\
\hline Hygienic disposal of stool & $918(46.9)$ & $913(47.6)$ & $5(11.2)$ & $<0.001$ & $1474(39.4)$ & 1459(39.5) & $15(33.2)$ & 0.45 \\
\hline Antenatal visit (4 plus) & $1565(78.2)$ & $1541(78.4)$ & $24(68.4)$ & 0.18 & $3480(87.0)$ & $3438(87.1)$ & $42(83.1)$ & 0.4 \\
\hline IPT-p & $830(41.0)$ & $812(40.9)$ & $18(46.2)$ & 0.55 & $2828(69.3)$ & 2793(69.3) & $35(70.3)$ & 0.90 \\
\hline Iron(90 day+) & $781(41.2)$ & $765(41.1)$ & $16(46.4)$ & 0.54 & $2266(59.3)$ & $2237(59.3)$ & $29(57.9)$ & 0.86 \\
\hline TT protected & $1416(70.9)$ & 1388(70.9) & $28(71.6)$ & 0.93 & $3099(79.0)$ & $3063(79.1)$ & $36(69.1)$ & 0.12 \\
\hline Health facility delivery & $1153(60.0)$ & 1132(59.9) & $21(63.3)$ & 0.71 & $2894(74.7)$ & $2861(74.8)$ & $33(64.7)$ & 0.16 \\
\hline Skilled delivery & $1183(61.5)$ & $1161(61.4)$ & $22(67.5)$ & 0.48 & $2926(75.4)$ & 2893(75.6) & $33(64.7)$ & 0.13 \\
\hline Contraceptives use & $468(23.3)$ & $461(23.4)$ & $7(21.1)$ & 0.77 & $1179(29.9)$ & $1169(28.0)$ & $10(21.6)$ & 0.30 \\
\hline Clean postnatal & $143(6.4)$ & $142(6.5)$ & $1(0.9)$ & NA & $1010(23.3)$ & $1000(23.4)$ & $10(13.4)$ & 0.07 \\
\hline Early breastfeeding & $1,082(51.7)$ & $1071(52.3)$ & $11(24.6)$ & 0.001 & $2245(54.8)$ & $2227(55.1)$ & $18(31.4)$ & 0.002 \\
\hline Household ITN/IRS & $1493(71.1)$ & $1467(71.5)$ & $26(59.1)$ & 0.12 & 3491 (83.6) & $3446(83.6)$ & $45(84.4)$ & 0.90 \\
\hline 8 interventions & $0(0.0)$ & $0(0.0)$ & $0(0.0)$ & NA & $58(2.1)$ & $58(2.1)$ & $0(0.0)$ & NA \\
\hline Total & $2045(100)$ & $2000(100)$ & $40(100)$ & & $4053(100)$ & $4000(100)$ & $53(100)$ & \\
\hline
\end{tabular}

$\mathrm{N}=$ total sample. $\mathrm{n}=$ number of observations. Eight interventions are antenatal visit, IPT-p, iron (90 day + ), tetanus toxoid vaccine, skilled delivery, clean postnatal care, early initiation of breastfeeding, and household ITN/IRS coverage. *Statistical significance at $95 \%$ confidence interval. Indoor Residual Spraying = ITN/IRS, Intermittent Preventive Treatment of Malaria in Pregnancy =IPT-p

Source: 2008 and 2014 Ghana Demographic and Health Surveys

Table 3 Interventions associated with under-five mortality

\begin{tabular}{llll}
\hline Intervention & Sample size & OR(95 \%Cl) & aOR (95 \%Cl) \\
\hline Early initiation of breastfeeding(within 1 h) & 6,072 & $0.32(0.19-0.54)$ & $0.38(0.21-0.69)$ \\
Clean postnatal care (within 2 days) & 5,768 & $0.32(0.16-0.65)$ & $0.34(0.14-0.82)$ \\
IPT-p & 5,965 & $1.21(0.49-3.00)$ & $1.64(0.76-3.54)$ \\
Skilled delivery & 6,040 & $1.10(0.55-2.20)$ & $1.77(0.60-5.25)$ \\
Tetanus toxoid vaccine & 5,980 & $0.82(0.47-1.40)$ & $0.86(0.41-1.79)$ \\
Hygienic disposal of stool & 6,043 & $0.43(0.21-0.88)$ & $0.74(0.33-1.66)$ \\
Iron intake(90days+) & 6,045 & $1.43(0.77-2.67)$ & $1.16(0.49-2.76)$ \\
Antenatal care (4+ visits) & 5,951 & $2.52(1.18-5.36)$ & $1.59(0.71-3.57)$ \\
\hline
\end{tabular}

Statistical significance with $95 \%$ confidence interval, unadjusted odds ratios (OR), and adjusted odds ratios (aOR). Intermittent Preventive Treatment of Malaria in Pregnancy $=$ IPT-p

Source: 2008 and 2014 Ghana Demographic and Health Surveys

with preceding birth intervals of less than two years twice more likely to die relative to those with longer birth interval $(\mathrm{aOR}=2.17,95 \% \mathrm{CI}: 1.07-4.40)$. On household factors, children in households with 3 or more children under-five years had their odds of death reduced by $81 \%$ $(\mathrm{aOR}=0.19,95 \% \mathrm{CI}: 0.07-0.54)$ compared to those in households with one or two children under five years old. Also, children in polygamous homes were 2 times more likely to die compared to children in monogamous homes (aOR $=1.94,95 \% \mathrm{CI}: 1.05-3.61)$. The odds of death was reduced by $45 \%$ among children whose mothers had health insurance coverage $(\mathrm{aOR}=0.55,95 \%$ CI: 0.31-0.96).

\section{Discussion}

Interventions and under-five mortality

Despite efforts at reducing U5M, mortality decline in Ghana is slow. Although interventions targeted at reducing under-five mortality might show efficacy under experimental conditions, their effectiveness might be suboptimal, resulting in their low impact on mortality. This study evaluated the effectiveness of the 
Table 4 Adjusted odds ratios of interventions with effect on under-five mortality, multiple logistic regression analysis after matching

\begin{tabular}{|c|c|c|c|c|c|c|c|c|}
\hline \multirow[t]{2}{*}{$\begin{array}{l}\text { Covariates } \\
\text { adjusted for } \\
\text { in the model }\end{array}$} & $\begin{array}{l}\text { Antenatal } \\
\text { care visits }\end{array}$ & $\begin{array}{l}\text { Skilled } \\
\text { delivery }\end{array}$ & Iron intake & $\begin{array}{l}\text { Early } \\
\text { initiation of } \\
\text { breastfeeding }\end{array}$ & $\begin{array}{l}\text { Clean } \\
\text { postnatal } \\
\text { care }\end{array}$ & IPT-P & $\begin{array}{l}\text { Hygienic } \\
\text { disposal } \\
\text { of stool }\end{array}$ & $\begin{array}{l}\text { Tetanus toxoid } \\
\text { vaccine }\end{array}$ \\
\hline & aOR $(95 \% \mathrm{Cl})$ & aOR (95 \%Cl) & aOR (95 \%Cl) & aOR (95 \%Cl) & aOR (95 \%Cl) & aOR (95 \%Cl) & aOR $(95 \% \mathrm{Cl})$ & aOR (95 \%Cl) \\
\hline \multicolumn{9}{|l|}{ Survey year } \\
\hline \multicolumn{9}{|l|}{2008} \\
\hline 2014 & $0.94(0.48-1.82)$ & $1.18(0.58-2.39)$ & $0.86(0.42-1.79)$ & $0.99(0.52-1.89)$ & $\begin{array}{l}0.73(0.36- \\
1.49)\end{array}$ & $\begin{array}{l}10.74(0.79- \\
3.82)\end{array}$ & $\begin{array}{l}1.41(0.56- \\
3.60)\end{array}$ & $0.99(0.53-1.85)$ \\
\hline \multicolumn{9}{|l|}{ Age(months) } \\
\hline \multicolumn{9}{|l|}{$<1$} \\
\hline 1 to 5 & $0.02(0.01-0.04)$ & $\begin{array}{l}0.01(0.003- \\
0.02)\end{array}$ & $\begin{array}{l}0.01(0.003- \\
0.03)\end{array}$ & $0.02(0.01-0.06)$ & $\begin{array}{l}0.01(0.004- \\
0.03)\end{array}$ & $\begin{array}{l}0.005(0.001- \\
0.02)\end{array}$ & $\begin{array}{l}0.01(0.005- \\
0.04)\end{array}$ & $0.02(0.01-0.04)$ \\
\hline 6 to 11 & $0.02(0.01-0.05)$ & $\begin{array}{l}0.01(0.003- \\
0.03)\end{array}$ & $\begin{array}{l}0.01(0.004- \\
0.03)\end{array}$ & $0.02(0.01-0.06)$ & $\begin{array}{l}0.01(0.002- \\
0.03)\end{array}$ & $\begin{array}{l}0.01(0.002- \\
0.03)\end{array}$ & $\begin{array}{l}0.01(0.004- \\
0.03)\end{array}$ & $0.02(0.01-0.05)$ \\
\hline$>=12$ months & $\begin{array}{l}0.005(0.002- \\
0.01)\end{array}$ & $\begin{array}{l}0.003(0.001- \\
0.01)\end{array}$ & $\begin{array}{l}0.004(0.001- \\
0.01)\end{array}$ & $0.01(0.003-0.02)$ & $\begin{array}{l}0.003(0.001- \\
0.01)\end{array}$ & $\begin{array}{l}0.002(0.001- \\
0.01)\end{array}$ & $\begin{array}{l}0.002(0.0005- \\
0.01)\end{array}$ & $0.01(0.002-0.01)$ \\
\hline \multicolumn{9}{|l|}{ Gender } \\
\hline \multicolumn{9}{|l|}{ Male } \\
\hline Female & $0.86(0.46-1.62)$ & $0.81(0.57-3.41)$ & $0.84(0.38-1.86)$ & $0.83(0.46-1.50)$ & $\begin{array}{l}0.85(0.40- \\
1.81)\end{array}$ & $\begin{array}{l}1.55(0.74- \\
3.28)\end{array}$ & $\begin{array}{l}1.04(0.47- \\
2.32)\end{array}$ & $0.84(0.46-1.52)$ \\
\hline \multicolumn{9}{|l|}{ Birth interval } \\
\hline \multicolumn{9}{|l|}{$>=2$ years } \\
\hline Less than 2 & $1.92(0.85-4.38)$ & $4.57(2.22-9.39)$ & $1.38(0.64-2.95)$ & $2.76(1.38-5.53)$ & $\begin{array}{l}2.91(1.43- \\
5.88)\end{array}$ & $\begin{array}{l}1.57(0.72- \\
3.43)\end{array}$ & $\begin{array}{l}1.49(0.61- \\
3.61)\end{array}$ & $2.22(1.09-4.51)$ \\
\hline \multicolumn{9}{|l|}{ Birth order } \\
\hline \multicolumn{9}{|l|}{ Below 3} \\
\hline$>=3$ & $1.65(0.79-3.45)$ & $2.41(0.98-5.90)$ & $1.05(0.47-2.34)$ & 1.85(0.88-3.89) & $\begin{array}{l}2.53(1.23- \\
5.19)\end{array}$ & $\begin{array}{l}1.93(0.87- \\
4.27)\end{array}$ & $\begin{array}{l}1.12(0.46- \\
2.73)\end{array}$ & $1.60(0.76-3.34)$ \\
\hline \multicolumn{9}{|l|}{ Birth weight } \\
\hline \multicolumn{9}{|l|}{ Normal } \\
\hline Small & $0.86(0.33-2.20)$ & $1.40(0.57-3.41)$ & NA & $0.81(0.27-2.40)$ & $\begin{array}{l}0.69(0.26- \\
1.83)\end{array}$ & NA & $\begin{array}{l}0.74(0.21- \\
2.54)\end{array}$ & $0.65(0.24-1.76)$ \\
\hline \multicolumn{9}{|l|}{ Multiple birth } \\
\hline \multicolumn{9}{|l|}{ No } \\
\hline Yes & $\begin{array}{l}7.08(1.41- \\
35.60)\end{array}$ & $1.08(0.15-7.94)$ & $\begin{array}{l}10.12(2.56- \\
40.00)\end{array}$ & $2.56(0.32-20.27)$ & $\begin{array}{l}1.69(0.33- \\
8.63)\end{array}$ & $\begin{array}{l}3.617(0.83- \\
15.63)\end{array}$ & $\begin{array}{l}43.64(12.24- \\
155.58)\end{array}$ & $7.48(1.81-30.98)$ \\
\hline \multicolumn{9}{|l|}{ Delivery type } \\
\hline \multicolumn{9}{|l|}{ Vaginal } \\
\hline Caesarean & $0.89(0.36-2.21)$ & $0.67(0.28-1.59)$ & $0.66(0.26-1.69)$ & $0.89(0.35-2.27)$ & $\begin{array}{l}0.52(0.19- \\
1.43)\end{array}$ & $\begin{array}{l}0.38(0.13- \\
1.08)\end{array}$ & $\begin{array}{l}0.48(0.16- \\
1.46)\end{array}$ & $0.83(0.32-2.14)$ \\
\hline \multicolumn{9}{|l|}{ Region } \\
\hline \multicolumn{9}{|l|}{ Northern belt } \\
\hline Middle belt & $0.80(0.33-1.94)$ & $0.61(0.23-1.56)$ & $1.20(0.46-3.18)$ & $0.59(0.25-1.39)$ & $\begin{array}{l}0.90(0.29- \\
2.75)\end{array}$ & $\begin{array}{l}0.62(0.21- \\
1.82)\end{array}$ & $\begin{array}{l}0.91(0.32- \\
2.58)\end{array}$ & $0.75(0.31-1.80)$ \\
\hline Southern belt & $0.36(0.12-1.09)$ & $0.42(0.13-1.39)$ & $0.63(0.18-2.19)$ & $0.45(0.16-1.28)$ & $\begin{array}{l}0.81(0.22- \\
3.06)\end{array}$ & $\begin{array}{l}0.28(0.07- \\
1.10)\end{array}$ & $\begin{array}{l}0.31(0.07- \\
1.32)\end{array}$ & $0.39(0.14-1.10)$ \\
\hline \multicolumn{9}{|l|}{$\begin{array}{l}\text { Urban/rural } \\
\text { residence }\end{array}$} \\
\hline \multicolumn{9}{|l|}{ Urban } \\
\hline Rural & $1.16(0.45-3.01)$ & $1.40(0.44-4.44)$ & $0.92(0.35-2.42)$ & $0.76(0.30-1.93)$ & $\begin{array}{l}0.83(0.32- \\
2.12)\end{array}$ & $\begin{array}{l}1.61(0.51- \\
5.11)\end{array}$ & $\begin{array}{l}2.02(0.52- \\
7.81)\end{array}$ & $1.09(0.39-3.05)$ \\
\hline \multicolumn{9}{|l|}{$\begin{array}{l}\text { Wealth } \\
\text { quintile }\end{array}$} \\
\hline Poorest & & & & & & & & \\
\hline
\end{tabular}


Table 4 (continued)

\begin{tabular}{|c|c|c|c|c|c|c|c|c|}
\hline \multirow[t]{2}{*}{$\begin{array}{l}\text { Covariates } \\
\text { adjusted for } \\
\text { in the model }\end{array}$} & \multirow{2}{*}{$\begin{array}{l}\text { Antenatal } \\
\text { care visits } \\
\text { aOR }(95 \% \mathrm{Cl})\end{array}$} & \multirow{2}{*}{$\begin{array}{l}\begin{array}{l}\text { Skilled } \\
\text { delivery }\end{array} \\
\text { aOR }(95 \% \mathrm{Cl})\end{array}$} & \multirow{2}{*}{$\begin{array}{l}\text { Iron intake } \\
\text { aOR }(95 \% \mathrm{Cl})\end{array}$} & \multirow{2}{*}{$\begin{array}{l}\text { Early } \\
\text { initiation of } \\
\text { breastfeeding } \\
\text { aOR }(95 \% \mathrm{Cl})\end{array}$} & \multirow{2}{*}{$\begin{array}{l}\text { Clean } \\
\text { postnatal } \\
\text { care } \\
\text { aOR }(95 \% \mathrm{Cl})\end{array}$} & \multirow{2}{*}{$\begin{array}{l}\text { IPT-P } \\
\text { aOR }(95 \% \mathrm{Cl})\end{array}$} & \multirow{2}{*}{$\begin{array}{l}\text { Hygienic } \\
\text { disposal } \\
\text { of stool } \\
\text { aOR }(95 \% \mathrm{Cl})\end{array}$} & \multirow{2}{*}{$\begin{array}{l}\text { Tetanus toxoic } \\
\text { vaccine } \\
\text { aOR }(95 \% \mathrm{Cl})\end{array}$} \\
\hline & & & & & & & & \\
\hline Poorer & $0.85(0.28-2.57)$ & $0.59(0.18-1.96)$ & $0.92(0.28-3.04)$ & $0.98(0.36-2.65)$ & $\begin{array}{l}1.52(0.37- \\
6.29)\end{array}$ & $0.91(0.28-3.00)$ & $\begin{array}{l}0.62(0.21- \\
1.83)\end{array}$ & $0.85(0.31-2.32)$ \\
\hline Middle & $1.32(0.41-4.23)$ & $1.57(0.45-5.49)$ & $1.20(0.33-4.33)$ & $1.15(0.32-4.09)$ & $\begin{array}{l}2.17(0.49- \\
9.68)\end{array}$ & $\begin{array}{l}4.67(1.14- \\
19.17)\end{array}$ & $\begin{array}{l}1.35(0.28- \\
6.54)\end{array}$ & $1.18(0.37-3.79)$ \\
\hline Richer & $0.79(0.16-4.00)$ & $1.47(0.26-8.48)$ & $1.06(0.22-5.18)$ & $0.86(0.18-4.03)$ & $\begin{array}{l}0.88(0.11- \\
7.07)\end{array}$ & $\begin{array}{l}1.09(0.16- \\
7.67)\end{array}$ & $\begin{array}{l}0.79(0.14- \\
4.63)\end{array}$ & $1.03(0.20-5.36)$ \\
\hline Richest & $1.35(0.23-7.73)$ & $\begin{array}{l}2.62(0.40- \\
17.16)\end{array}$ & $1.24(0.23-6.75)$ & $1.75(0.30-10.08)$ & $\begin{array}{l}3.85(0.34- \\
43.59)\end{array}$ & $\begin{array}{l}3.61(0.42- \\
22.21)\end{array}$ & $\begin{array}{l}1.15(0.15- \\
8.76)\end{array}$ & $1.64(0.25-10.60)$ \\
\hline \multicolumn{9}{|l|}{$\begin{array}{l}\text { Polygamous } \\
\text { home }\end{array}$} \\
\hline \multicolumn{9}{|l|}{$\begin{array}{l}\text { Not polyga- } \\
\text { mous }\end{array}$} \\
\hline Polygamous & $2.22(1.15-4.28)$ & $2.14(1.01-4.51)$ & 1.64(0.73-3.70) & $2.82(1.53-5.17)$ & $\begin{array}{l}3.16(1.44- \\
6.95)\end{array}$ & $\begin{array}{l}1.52(0.70- \\
3.33)\end{array}$ & $\begin{array}{l}0.94(0.29- \\
3.05)\end{array}$ & $1.95(1.06-3.60)$ \\
\hline \multicolumn{9}{|l|}{$\begin{array}{l}\text { Household } \\
\text { size }\end{array}$} \\
\hline \multicolumn{9}{|l|}{$<6$} \\
\hline$>=6$ & $1.57(0.72-3.41)$ & $1.49(0.68-3.27)$ & $2.12(0.96-4.68)$ & $1.22(0.56-2.68)$ & $\begin{array}{l}1.60(0.77- \\
3.36)\end{array}$ & $\begin{array}{l}2.47(1.12- \\
5.43)\end{array}$ & $\begin{array}{l}1.31(0.60- \\
2.84)\end{array}$ & $1.24(0.58-2.67)$ \\
\hline \multicolumn{9}{|l|}{$\begin{array}{l}\text { No. of CU5 in } \\
\text { household }\end{array}$} \\
\hline \multicolumn{9}{|l|}{$1-2$} \\
\hline 3 and above & $0.18(0.06-0.53)$ & $0.12(0.03-0.44)$ & $0.16(0.05-0.51)$ & $0.15(0.05-0.50)$ & $\begin{array}{l}0.07(0.02- \\
0.23)\end{array}$ & $\begin{array}{l}0.17(0.06- \\
0.44)\end{array}$ & $\begin{array}{l}0.15(0.04- \\
0.55)\end{array}$ & $0.20(0.07-0.54)$ \\
\hline \multicolumn{9}{|l|}{ Maternal age } \\
\hline \multicolumn{9}{|l|}{$<35$} \\
\hline$>=35$ & $0.67(0.30-1.51)$ & $0.60(0.24-1.48)$ & $1.03(0.46-2.33)$ & $0.57(0.24-1.32)$ & $\begin{array}{l}0.54(0.21- \\
1.35)\end{array}$ & $\begin{array}{l}0.39(0.18- \\
0.84)\end{array}$ & $0.34(0.11-1.00)$ & $0.73(0.35-1.55)$ \\
\hline \multicolumn{9}{|l|}{$\begin{array}{l}\text { Maternal } \\
\text { education }\end{array}$} \\
\hline \multicolumn{9}{|l|}{ None } \\
\hline Primary & $1.38(0.54-3.52)$ & $1.45(0.52-4.05)$ & $2.13(0.75-6.05)$ & $1.52(0.59-3.93)$ & $\begin{array}{l}1.39(0.58- \\
3.32)\end{array}$ & $\begin{array}{l}0.72(0.27- \\
1.88)\end{array}$ & $\begin{array}{l}1.33(0.52- \\
3.39)\end{array}$ & $1.06(0.43-2.66)$ \\
\hline $\begin{array}{l}\text { Secondary or } \\
\text { higher }\end{array}$ & $0.59(0.21-1.65)$ & $0.83(0.28-2.46)$ & $0.71(0.26-1.91)$ & $0.57(0.21-1.59)$ & $\begin{array}{l}0.84(0.34- \\
2.10)\end{array}$ & $\begin{array}{l}0.34(0.12- \\
0.98)\end{array}$ & $\begin{array}{l}0.63(0.19- \\
2.05)\end{array}$ & $0.49(0.19-1.28)$ \\
\hline \multicolumn{9}{|l|}{ Married } \\
\hline \multicolumn{9}{|l|}{ Not Married } \\
\hline Married & $0.70(0.32-1.53)$ & $0.38(0.17-0.87)$ & $0.73(0.29-1.82)$ & $0.64(0.31-1.35)$ & $\begin{array}{l}0.67(0.31- \\
1.46)\end{array}$ & $\begin{array}{l}0.71(0.32- \\
1.59)\end{array}$ & $\begin{array}{l}0.71(0.27- \\
1.86)\end{array}$ & $0.71(0.34-1.47)$ \\
\hline \multicolumn{9}{|l|}{ Religion } \\
\hline \multicolumn{9}{|l|}{ Orthodox } \\
\hline Pentecostal & $0.85(0.37-1.95)$ & $1.41(0.52-3.79)$ & $0.61(0.22-1.67)$ & $1.13(0.49-2.61)$ & $\begin{array}{l}0.94(0.38- \\
2.33)\end{array}$ & $\begin{array}{l}0.72(0.27- \\
1.95)\end{array}$ & $\begin{array}{l}0.58(0.22- \\
1.54)\end{array}$ & $0.81(0.37-1.78)$ \\
\hline Islam & $1.30(0.48-3.51)$ & $\begin{array}{l}3.37(1.06- \\
10.73)\end{array}$ & $1.22(0.36-4.13)$ & $2.13(0.79-5.77)$ & $\begin{array}{l}3.81(1.27- \\
11.39)\end{array}$ & $\begin{array}{l}2.59(0.95- \\
7.10)\end{array}$ & $\begin{array}{l}0.61(0.18- \\
2.09)\end{array}$ & $1.35(0.54-3.41)$ \\
\hline Others & $1.17(0.37-3.70)$ & $\begin{array}{l}4.14(0.94- \\
18.20)\end{array}$ & $0.93(0.19-4.45)$ & $2.17(0.67-7.06)$ & $\begin{array}{l}1.90(0.52- \\
6.98)\end{array}$ & $\begin{array}{l}2.66(0.77- \\
9.25)\end{array}$ & $\begin{array}{l}0.98(0.30- \\
3.16)\end{array}$ & $1.31(0.41-4.15)$ \\
\hline \multicolumn{9}{|l|}{ Ethnicity } \\
\hline \multicolumn{9}{|l|}{ Akan } \\
\hline Mole-Dagbani & $0.56(0.15-2.01)$ & $1.01(0.27-3.85)$ & $0.79(0.23-2.75)$ & $0.48(0.15-1.52)$ & $\begin{array}{l}0.53(0.15- \\
1.91)\end{array}$ & $\begin{array}{l}0.50(0.12- \\
2.07)\end{array}$ & $\begin{array}{l}1.29(0.38- \\
4.42)\end{array}$ & $0.65(0.20-2.05)$ \\
\hline
\end{tabular}


Table 4 (continued)

\begin{tabular}{|c|c|c|c|c|c|c|c|c|}
\hline \multirow[t]{2}{*}{$\begin{array}{l}\text { Covariates } \\
\text { adjusted for } \\
\text { in the model }\end{array}$} & \multirow{2}{*}{$\begin{array}{l}\text { Antenatal } \\
\text { care visits } \\
\text { aOR }(95 \% \mathrm{Cl})\end{array}$} & \multirow{2}{*}{$\begin{array}{l}\begin{array}{l}\text { Skilled } \\
\text { delivery }\end{array} \\
\text { aOR }(95 \% \mathrm{Cl})\end{array}$} & \multirow{2}{*}{$\begin{array}{l}\text { Iron intake } \\
\text { aOR }(95 \% \mathrm{Cl})\end{array}$} & \multirow{2}{*}{$\begin{array}{l}\text { Early } \\
\text { initiation of } \\
\text { breastfeeding } \\
\text { aOR }(95 \% \mathrm{Cl})\end{array}$} & \multirow{2}{*}{$\begin{array}{l}\text { Clean } \\
\text { postnatal } \\
\text { care } \\
\text { aOR }(95 \% \mathrm{Cl})\end{array}$} & \multirow{2}{*}{$\begin{array}{l}\text { IPT-P } \\
\text { aOR }(95 \% \mathrm{Cl})\end{array}$} & \multirow{2}{*}{$\begin{array}{l}\text { Hygienic } \\
\text { disposal } \\
\text { of stool } \\
\text { aOR }(95 \% \mathrm{Cl})\end{array}$} & \multirow{2}{*}{$\begin{array}{l}\text { Tetanus toxoic } \\
\text { vaccine } \\
\text { aOR }(95 \% \mathrm{Cl})\end{array}$} \\
\hline & & & & & & & & \\
\hline Others & $1.26(0.54-2.95)$ & $1.20(0.52-2.78)$ & $1.53(0.53-4.37)$ & $0.86(0.40-1.86)$ & $\begin{array}{l}1.78(0.77- \\
4.12)\end{array}$ & $\begin{array}{l}1.03(0.41- \\
2.54)\end{array}$ & $\begin{array}{l}1.22(0.48- \\
3.08)\end{array}$ & $1.30(0.58-2.93)$ \\
\hline \multicolumn{9}{|l|}{$\begin{array}{l}\text { Mother's } \\
\text { employment }\end{array}$} \\
\hline \multicolumn{9}{|l|}{ Not employed } \\
\hline Employed & $0.67(0.29-1.54)$ & $0.46(0.21-1.01)$ & $0.64(0.27-1.51)$ & $0.90(0.40-2.04)$ & $\begin{array}{l}1.41(0.53- \\
3.73)\end{array}$ & $\begin{array}{l}0.84(0.36- \\
1.97)\end{array}$ & $\begin{array}{l}0.77(0.28- \\
2.11)\end{array}$ & $0.74(0.34-1.65)$ \\
\hline \multicolumn{9}{|l|}{$\begin{array}{l}\text { Contracep- } \\
\text { tives use }\end{array}$} \\
\hline \multicolumn{9}{|l|}{ No use } \\
\hline Use & $1.19(0.51-2.78)$ & $1.36(0.54-3.44)$ & $0.89(0.36-2.23)$ & $1.40(0.62-3.14)$ & $\begin{array}{l}1.52(0.62- \\
3.72)\end{array}$ & $\begin{array}{l}0.90(0.41- \\
1.97)\end{array}$ & $\begin{array}{l}1.07(0.29- \\
3.88)\end{array}$ & $1.43(0.64-3.16)$ \\
\hline \multicolumn{9}{|l|}{ Sanitation } \\
\hline \multicolumn{9}{|l|}{ Not improved } \\
\hline Improved & $1.05(0.40-2.74)$ & $1.05(0.37-3.02)$ & $0.60(0.21-1.72)$ & $0.95(0.23-3.98)$ & $\begin{array}{l}0.48(0.12- \\
1.88)\end{array}$ & $\begin{array}{l}0.65(0.19- \\
2.15)\end{array}$ & $\begin{array}{l}0.88(0.30- \\
2.58)\end{array}$ & $0.90(0.33-2.42)$ \\
\hline \multicolumn{9}{|l|}{$\begin{array}{l}\text { Antenatal } \\
\text { visits }\end{array}$} \\
\hline \multicolumn{9}{|l|}{$<4$ plus visits } \\
\hline $4+$ visit & $1.59(0.71-3.57)$ & $1.22(0.47-3.15)$ & $0.88(0.26-2.96)$ & $0.84(0.41-1.72)$ & $\begin{array}{l}0.91(0.37- \\
2.24)\end{array}$ & $\begin{array}{l}2.05(0.69- \\
6.10)\end{array}$ & $\begin{array}{l}0.99(0.39- \\
2.50)\end{array}$ & $0.88(0.40-1.91)$ \\
\hline \multicolumn{9}{|l|}{$\begin{array}{l}\text { Tetanus toxoid } \\
\text { vaccine }\end{array}$} \\
\hline \multicolumn{9}{|l|}{ Not received } \\
\hline Received & $0.85(0.37-1.94)$ & $0.59(0.26-1.33)$ & $1.53(0.57-4.11)$ & $0.57(0.26-1.25)$ & $\begin{array}{l}0.49(0.23- \\
1.05)\end{array}$ & $\begin{array}{l}0.58(0.23- \\
1.35)\end{array}$ & $\begin{array}{l}2.06(0.91- \\
4.69)\end{array}$ & $0.86(0.41-1.79)$ \\
\hline \multicolumn{9}{|l|}{ Skilled delivery } \\
\hline \multicolumn{9}{|l|}{ Not skilled } \\
\hline Skilled & $1.48(0.54-4.03)$ & $1.77(0.60-5.25)$ & $1.71(0.61-4.77)$ & $1.58(0.61-4.11)$ & $\begin{array}{l}1.58(0.51- \\
4.91)\end{array}$ & $\begin{array}{l}2.01(0.69- \\
5.83)\end{array}$ & $\begin{array}{l}0.94(0.32- \\
2.80)\end{array}$ & $1.70(0.68-4.24)$ \\
\hline \multicolumn{9}{|l|}{$\begin{array}{l}\text { Improved } \\
\text { water source }\end{array}$} \\
\hline \multicolumn{9}{|l|}{ Not improved } \\
\hline Improved & $1.40(0.61-3.21)$ & $1.39(0.56-3.42)$ & $2.66(0.86-8.23)$ & $1.34(0.63-2.82)$ & $\begin{array}{l}1.33(0.56- \\
3.16)\end{array}$ & $\begin{array}{l}1.76(0.69- \\
4.65)\end{array}$ & $\begin{array}{l}0.98(0.39- \\
2.46)\end{array}$ & $1.31(0.61-2.82)$ \\
\hline \multicolumn{9}{|l|}{$\begin{array}{l}\text { Water con- } \\
\text { nected }\end{array}$} \\
\hline \multicolumn{9}{|l|}{$\begin{array}{l}\text { Not con- } \\
\text { nected }\end{array}$} \\
\hline Connected & $0.77(0.21-2.78)$ & $0.48(0.13-1.88)$ & $0.58(0.11-2.94)$ & $0.52(0.14-1.96)$ & $\begin{array}{l}0.84(0.18- \\
3.85)\end{array}$ & $\begin{array}{l}0.46(0.08- \\
2.72)\end{array}$ & $\begin{array}{l}0.42(0.09- \\
1.95)\end{array}$ & $0.64(0.17-2.38)$ \\
\hline \multicolumn{9}{|l|}{$\begin{array}{l}\text { ITN/IRS protec- } \\
\text { tion }\end{array}$} \\
\hline \multicolumn{9}{|l|}{ Not protected } \\
\hline Protected & $0.83(0.37-1.87)$ & $0.86(0.32-2.35)$ & $1.02(0.42-2.50)$ & $0.79(0.37-1.68)$ & $\begin{array}{l}0.76(0.38- \\
1.53)\end{array}$ & $\begin{array}{l}1.25(0.41- \\
3.75)\end{array}$ & $\begin{array}{l}0.37(0.16- \\
0.87)\end{array}$ & $0.79(0.37-1.72)$ \\
\hline \multicolumn{9}{|l|}{$\begin{array}{l}\text { Hygienic dis- } \\
\text { posal of stool }\end{array}$} \\
\hline Not hygienic & & & & & & & & \\
\hline Hygienic & $0.54(0.28-1.05)$ & $0.63(0.30-1.32)$ & $0.56(0.26-1.18)$ & $0.58(0.31-1.06)$ & $\begin{array}{l}0.52(0.24- \\
1.11)\end{array}$ & $\begin{array}{l}0.43(0.20- \\
0.91)\end{array}$ & $\begin{array}{l}0.74(0.33- \\
1.66)\end{array}$ & $0.62(0.33-1.15)$ \\
\hline
\end{tabular}


Table 4 (continued)

\begin{tabular}{|c|c|c|c|c|c|c|c|c|}
\hline \multirow[t]{2}{*}{$\begin{array}{l}\text { Covariates } \\
\text { adjusted for } \\
\text { in the model }\end{array}$} & $\begin{array}{l}\text { Antenatal } \\
\text { care visits }\end{array}$ & $\begin{array}{l}\text { Skilled } \\
\text { delivery }\end{array}$ & Iron intake & $\begin{array}{l}\text { Early } \\
\text { initiation of } \\
\text { breastfeeding }\end{array}$ & $\begin{array}{l}\text { Clean } \\
\text { postnatal } \\
\text { care }\end{array}$ & IPT-P & $\begin{array}{l}\text { Hygienic } \\
\text { disposal } \\
\text { of stool }\end{array}$ & $\begin{array}{l}\text { Tetanus toxoid } \\
\text { vaccine }\end{array}$ \\
\hline & aOR (95 \%Cl) & aOR (95 \%Cl) & aOR (95 \%Cl) & aOR (95 \%Cl) & aOR (95 \%Cl) & aOR (95 \%Cl) & aOR $(95 \% \mathrm{Cl})$ & aOR (95 \%Cl) \\
\hline \multicolumn{9}{|l|}{$\begin{array}{l}\text { Early breast- } \\
\text { feeding }\end{array}$} \\
\hline \multicolumn{9}{|l|}{$\begin{array}{l}\text { Not early } \\
\text { initiation }\end{array}$} \\
\hline Early initiation & $0.37(0.19-0.73)$ & $0.32(0.14-0.72)$ & $0.41(0.17-0.99)$ & $0.38(0.21-0.69)$ & $\begin{array}{l}0.57(0.28- \\
1.18)\end{array}$ & $\begin{array}{l}0.21(0.10- \\
0.46)\end{array}$ & $\begin{array}{l}0.29(0.11- \\
0.77)\end{array}$ & $0.42(0.22-0.79)$ \\
\hline \multicolumn{9}{|l|}{$\begin{array}{l}\text { Clean post- } \\
\text { natal care in } \\
2 \text { days }\end{array}$} \\
\hline \multicolumn{9}{|l|}{$\begin{array}{l}\text { No clean } \\
\text { postnatal }\end{array}$} \\
\hline $\begin{array}{l}\text { Clean post- } \\
\text { natal }\end{array}$ & $0.29(0.11-0.75)$ & $0.20(0.05-0.76)$ & $0.24(0.06-1.03)$ & $0.43(0.19-0.97)$ & $\begin{array}{l}0.34(0.14- \\
0.82)\end{array}$ & $\begin{array}{l}0.15(0.05- \\
0.46)\end{array}$ & $\begin{array}{l}0.27(0.05- \\
1.46)\end{array}$ & $0.40(0.16-1.01)$ \\
\hline \multicolumn{9}{|l|}{$\begin{array}{l}\text { Iron } \\
\text { intake > } 90 \\
\text { days }\end{array}$} \\
\hline \multicolumn{9}{|l|}{ No iron intake } \\
\hline Iron intake & $1.51(0.73-3.11)$ & $1.30(0.62-2.72)$ & $1.16(0.49-2.76)$ & $1.31(0.64-2.65)$ & $\begin{array}{l}1.33(0.58- \\
3.09)\end{array}$ & $\begin{array}{l}1.62(0.79- \\
3.30)\end{array}$ & $\begin{array}{l}2.26(0.83- \\
6.17)\end{array}$ & $1.51(0.73-3.10)$ \\
\hline \multicolumn{9}{|l|}{ IPT-p } \\
\hline \multicolumn{9}{|l|}{ No IPT-p } \\
\hline IPT-p & $1.65(0.76-3.57)$ & $2.36(1.14-4.89)$ & $1.74(0.78-3.92)$ & $1.74(0.89-3.39)$ & $\begin{array}{l}1.20(0.65- \\
2.22)\end{array}$ & $\begin{array}{l}1.74(0.79- \\
3.82)\end{array}$ & $\begin{array}{l}0.81(0.35- \\
1.86)\end{array}$ & $1.47(0.74-2.94)$ \\
\hline \multicolumn{9}{|l|}{ NHIS status } \\
\hline \multicolumn{9}{|l|}{ Not covered } \\
\hline Covered & $0.50(0.27-0.91)$ & $0.51(0.27-0.98)$ & $0.68(0.33-1.38)$ & $0.68(0.38-1.22)$ & $\begin{array}{l}0.33(0.14- \\
0.75)\end{array}$ & $\begin{array}{l}0.55(0.25- \\
1.22)\end{array}$ & $\begin{array}{l}0.74(0.34- \\
1.61)\end{array}$ & $0.51(0.28-0.94)$ \\
\hline
\end{tabular}

NB: Not applicable. The factor is an intermediate in the causal pathway and was not adjusted for in the model. Statistical significance with $95 \%$ confidence interval, unadjusted odds ratios (OR), and adjusted odds ratios (aOR)

various child health interventions on U5M in Ghana. The results showed that the various child health interventions impact child mortality differently. Considering that increase in coverage of interventions is the main strategy to address U5M and possibly achieve the SDG 3 target 2, the lack of effect of key interventions on U5M implies that an increase in coverage alone might not yield the needed decline in mortality to achieve the SDG target.

Children who had clean postnatal care had a $66 \%$ reduced average odds of death. Clean postnatal care is defined as neonates receiving a preventive postnatal visit within $48 \mathrm{~h}$ of delivery [3]. The assumption is that neonates who receive clean postnatal care will subsequently receive adequate clean postnatal care in the home [3, 38]. Sepsis is a major cause of neonatal mortality, and cord care influences the incidence of neonatal sepsis. Therefore, clean birth and postnatal care practices are recommended for reducing infection and neonatal mortality [39], and thus, the recommendation that women after delivery have postnatal care within $48 \mathrm{~h}$. This will ensure that infections are identified early for timely management.

Clean postnatal care can significantly contribute to the elimination of neonatal tetanus which is a significant contributor to neonatal mortality[40]. From the 2014 Ghana Demographic and Health Survey, mothers received clean postnatal care from skilled or traditional birth attendants. Skilled health workers include doctors, nurses, midwives, community health nurses, or community health officers. Clean postnatal care is one of the interventions with the lowest coverage levels in Ghana. Coverage in 2008 was $6.5 \%$, while that in 2014 was $22.8 \%$ [11]. Its low coverage is, therefore, an avenue to further reduce deaths if its coverage level is increased. The result of the protective effect of clean postnatal care on mortality in this study is similar to that documented [5, 41-43].

Breastfeeding has been shown to reduce the risk of infections and, consequently, the death of children under-five years old [41, 44-47]. Early breastfeeding has 
Table 5 Factors associated with under-five mortality among children 0-59 months without matching $(N=6,098)$

\begin{tabular}{|c|c|c|c|c|c|}
\hline children $0-59$ months & Та, С III & & Characteristics & OR (95\% CI) & aOR(95\% Cl) \\
\hline Characteristics & OR $(95 \% \mathrm{Cl})$ & aOR(95 \% Cl) & $<35$ & & \\
\hline Survey year & & & $>=35$ & $1.19(0.73-1.93)$ & $0.81(0.38-1.71)$ \\
\hline 2008 & & & Maternal education & & \\
\hline 2014 & $0.63(0.40-1.0)$ & $0.89(0.49-1.62)$ & None & & \\
\hline Age(months) & & & Primary & $1.44(0.82-2.53)$ & $1.16(0.48-2.80)$ \\
\hline$<1$ & & & Secondary or higher & $0.36(0.20-0.65)$ & $0.51(0.20-1.30)$ \\
\hline 1 to 5 & $0.02(0.01-0.05)$ & $0.02(0.01-0.04)$ & Married & & \\
\hline 6 to 11 & $0.03(0.01-0.06)$ & $0.02(0.01-0.05)$ & Not Married & & \\
\hline 12 and above & $0.01(0.004-0.02$ & $0.01(0.002-0.01)$ & Married & $0.92(0.56-1.50)$ & $0.69(0.34-1.39)$ \\
\hline Gender & & & Religion & & \\
\hline Male & & & Orthodox & & \\
\hline Female & $0.90(0.57-1.41)$ & $0.87(0.49-1.56)$ & Pentecostal & $0.83(0.45-1.55)$ & $0.90(0.40-2.01)$ \\
\hline Birth interval & & & Islam & $1.35(0.67-2.72)$ & $1.32(0.52-3.34)$ \\
\hline$>=2$ years & & & Others & $2.02(0.90-4.55)$ & $1.26(0.41-3.86)$ \\
\hline Less than 2 & $1.88(0.94-3.76)$ & $2.17(1.07-4.40)$ & Ethnicity & & \\
\hline Birth order & & & Akan & & \\
\hline Below 3 & & & Mole-Dagbani & $1.35(0.74-2.45)$ & $0.66(0.21-2.08)$ \\
\hline$>=3$ & $1.95(1.21-3.14)$ & $1.80(0.87-3.68)$ & Others & $1.39(0.80-2.40)$ & $1.25(0.57-2.73)$ \\
\hline Birth weight & & & Mother's employment & & \\
\hline Normal & & & Not employed & & \\
\hline Small & $1.26(0.67-2.37)$ & $0.84(0.32-2.24)$ & Employed & $0.76(0.42-1.37)$ & $0.76(0.34-1.72)$ \\
\hline Multiple birth & & & Contraceptives use & & \\
\hline Singleton & & & No use & & \\
\hline Multiple birth & $4.99(2.20-11.33)$ & $6.55(1.56-27.57)$ & Use & $0.71(0.38-1.30)$ & $1.40(0.63-3.08)$ \\
\hline Delivery type & & & Sanitation & & \\
\hline Vaginal & & & Not improved & & \\
\hline Caesarean & $1.09(0.50-2.41)$ & $0.87(0.35-2.17)$ & Improved & $0.64(0.29-1.38)$ & $0.91(0.35-2.36)$ \\
\hline Region & & & Antenatal visits & & \\
\hline Northern belt & & & $<4$ plus visits & & \\
\hline Middle belt & $0.74(0.43-1.28)$ & $0.75(0.32-1.75)$ & $4+$ visit & $0.61(0.36-1.04)$ & $0.82(0.40-1.69)$ \\
\hline Southern belt & $0.46(0.26-0.83)$ & $0.44(0.16-1.23)$ & Tetanus toxoid vaccine & & \\
\hline Urban/rural residence & & & Not received & & \\
\hline Urban & & & Received & $0.73(0.44-1.21)$ & $0.83(0.40-1.75)$ \\
\hline Rural & $1.34(0.82-2.18)$ & $0.86(0.35-2.16)$ & Skilled delivery & & \\
\hline Wealth quintile & & & Not skilled & & \\
\hline Poorest & & & Skilled & $0.80(0.49-1.32)$ & $1.57(0.64-3.89)$ \\
\hline Poorer & $0.70(0.37-1.33)$ & $0.86(0.32-2.32)$ & Improved water source & & \\
\hline Middle & $0.59(0.29-1.19)$ & $1.00(0.32-3.18)$ & Not improved & & \\
\hline Richer & $0.58(0.27-1.22)$ & $0.82(0.19-3.57)$ & Improved & $1.45(0.82-2.55)$ & $1.40(0.66-2.98)$ \\
\hline Richest & $0.37(0.15-0.94)$ & $1.21(0.24-6.21)$ & Water connected & & \\
\hline Polygamous home & & & Not connected & & \\
\hline Not polygamous & & & Connected & $0.89(0.35-2.26)$ & $0.65(0.19-2.24)$ \\
\hline Polygamous & $1.99(1.14-3.49)$ & $1.94(1.05-3.61)$ & ITN/IRS protection & & \\
\hline Household size & & & Not protected & & \\
\hline$<6$ & & & Protected & $0.70(0.41-1.20)$ & $0.77(0.37-1.60)$ \\
\hline$>=6$ & $1.69(1.05-2.72)$ & $1.01(0.51-2.36)$ & Hygienic disposal of stool & & \\
\hline No. of CU5 in household & & & Not hygienic & & \\
\hline $1-2$ & & & Hygienic & $0.35(0.20-0.62)$ & $0.59(0.32-1.07)$ \\
\hline 3 and above & $1.03(0.52-2.04)$ & $0.19(0.07-0.54)$ & Clean postnatal care in 2 days & & \\
\hline
\end{tabular}

Table 5 (continued)

\section{Characteristics}

$<35$

$1.44(0.82-2.53)$

$1.16(0.48-2.80)$

Primary

Married

Not Married

Pentecostal

$0.90(0.40-2.01)$

$.32(0.52-3.34)$

Islam

Ethnicity

Mole-Dagbani

$1.35(0.74-2.45)$

$0.66(0.21-2.08)$

Mother's employment

Contraceptives use

\author{
Page 13 of 17
}


Table 5 (continued)

\begin{tabular}{lll}
\hline Characteristics & OR $(95 \% \mathrm{Cl})$ & aOR $(95 \% \mathrm{Cl})$ \\
\hline $\begin{array}{l}\text { No clean postnatal } \\
\text { Clean postnatal }\end{array}$ & $0.36(0.18-0.71)$ & $0.40(0.16-0.99)$ \\
Iron intake $>90$ days & & \\
No iron intake & & \\
Iron intake & $1.01(0.63-1.61)$ & $1.49(0.75-2.94)$ \\
Early breastfeeding & & \\
Not early & $0.33(0.2-0.54)$ & $0.39(0.21-0.72)$ \\
Early & & \\
IPT-p & & \\
No IPT-p & $1.00(0.62-1.63)$ & $1.47(0.76-2.86)$ \\
IPT-p & & \\
NHIS status & & \\
Not covered & & \\
Covered & $0.70(0.44-1.13)$ & $0.55(0.31-0.96)$ \\
\hline
\end{tabular}

Statistical significance with $95 \%$ confidence interval, unadjusted odds ratios (OR), and adjusted odds ratios (aOR)

additional benefits as it promotes warmth and bonding between infants and their mothers. In this study, early initiation of breastfeeding was associated with a $62 \%$ reduction in the average odds of death. Similar results of the association between early initiation of breastfeeding and under-five mortality have been documented [44-48]. According to the GDHS, the coverage level of early initiation of breastfeeding was $25.5 \%$ in $1998,46.3 \%$ in 2003 , $52.3 \%$ in 2008 , and $55.6 \%$ in 2014 [11,38]. This trend of coverage increase is slow. Considering the 2014 coverage level of the intervention, it has the potential to further reduced mortality if its coverage level is increased. Since this intervention does not require much logistics or expenditure to implement compared to interventions like skilled delivery, it should be prioritized.

The lack of effect of the other interventions on underfive mortality could be attributed to the context of the implementation of these interventions. The context could include the incidence of the disease(s) each intervention is targeted at and the quality of the implementation of the interventions. There is evidence of poor quality of skilled delivery contributing to lack of effect of skilled delivery on child mortality [49]. Disparities in the association of skilled delivery with neonatal mortality have been documented in different geographic areas. While skilled delivery improved neonatal mortality in Latin America and the Caribbean, it was associated with worse neonatal mortality in Africa [50]. The quality and availability of logistics and health personnel for skilled delivery might explain the differences in the effectiveness of skilled delivery in different places [50].

Low incidence of disease that the intervention is targeted at could also explain the lack of statistical significance of the association between interventions and under-five mortality [51]. With a low incidence of disease, the intervention will have fewer deaths to prevent and, therefore, low effectiveness. That could be the case for the tetanus toxoid vaccine. In addition, improvement in birthing practices could reduce infections due to Clostridium tetani, and therefore, low risk of neonatal tetanus, a resultant reduced risk of death from neonatal tetanus. Currently, Ghana is at the elimination state of neonatal tetanus, which means a lower risk of neonatal tetanus infection [51].

With multiple interventions which have a direct and indirect effect on under-five mortality been implemented in the midst of socio-economic factors that also affect under-five mortality, Chowdhury [52] observed that proximate factors have a stronger effect on under-five mortality than more distal factors. Iron intake, antenatal care visits, and intermittent preventive treatment of malaria in pregnancy (IPT-p) have a relatively indirect effect on U5M and, therefore, could account for their lack of statistically significant effect on mortality.

\section{Other factors and under-five mortality}

In the midst of interventions, some socio-demographic factors remained associated with U5M. Children in households with three or more children under-five years were $81 \%$ less likely to die than those with one or two children. Having more children under-five years could mean the mother will have more experience taking care of children, including identifying signs and symptoms of diseases. More children under-five years in a household have been associated with early careseeking in Niger [53]. Children in households with a higher number of members also had reduced odds of neonatal and under-five mortality in Ghana [33].

Health insurance membership offers financial access to healthcare and is associated with increased and timely healthcare-seeking [54-56]. It could therefore increase the use of all the healthcare-associated interventions, and thus, its association with a $45 \%$ reduction in the odds of death of children under-five years in this study. A similar result of the protective effect of NHIS on mortality has been reported [33].

The higher odds of death of younger children and multiple births have been documented [41]. In this study, while multiple births were about 7 times more likely to die, compared to neonates, odds of deaths was reduced by about $99 \%$ among children 12 months and older. Addressing the increased odds of death with multiple births and younger children will require improved access to quality skilled delivery. Unfortunately, skilled delivery was not associated with mortality reduction. Younger children, especially neonates, 
are less developed and more susceptible to infections. However, specialized and advanced care needed during the neonatal period is unavailable, especially in poverty-ridden communities. Considering the causes of neonatal deaths such as sepsis, diarrhoea, pneumonia, and asphyxia, quality skilled delivery, improved nutrition, and improved hygiene will play essential roles in its reduction [57]. But, coverages of hygiene and sanitation interventions are among the interventions with the lowest coverage levels in Ghana [3, 38]. Also, coverage levels of exclusive breastfeeding and complementary feeding are on the decline $[3,38]$.

Additionally, children from mothers in polygamous marriages had twice higher odds of death. This finding is consistent with results reported in other studies [58, 59]. Rivalry among wives was attributed to the higher odds of death. Limited resources and overcrowding have also been cited as reasons for the positive correlation of polygamy and child mortality [60].

Lastly, children of birth intervals of less than two years also had twice higher odds of death compared to those of longer intervals. With shorter birth intervals, mothers might not have replenished nutrients used during the previous pregnancy resulting in under-nutrition [61]. After delivery, there will be competition for maternal resources, including time to care for the children. This can compromise the quality of their care resulting in infections, improper nutrition, and poor health. A shorter birth interval will also limit the duration of breastfeeding, which can affect the development of the older child. Similar results on the higher risk of death among children with shorter birth intervals have been documented in Ghana [5, 62].

\section{Limitations and strength of the study}

On limitations, exact matching coarsened like other matching methods matches on measured variables (potential confounders) contained in the data set, unlike randomization that controls for measured and unmeasured potential confounders. However, balanced was achieved between the treated and untreated groups using the variables available in the data set. Additionally, potential confounders adjusted for in this multiple regression analysis comprised all measured potential confounders, and therefore, the effect of unobserved variables is ignorable.

Also, $48 \%$ of observations had at least one missing value. Multiple imputation was done to address the missing data. On the strengths of the study, nationally representative data were used, which had high response rates (over $90 \%$ ), and therefore, the study findings are generalizable. The pooling of the data also increased the power of the study and, thus, the validity of the results. The matching method that was used reduced model dependence and thus, increases the robustness of the analysis. Lastly, the restriction of the analysis to only the last births reduced the influence of recall bias on the study results.

\section{Conclusions}

Of the eight (8) interventions assessed for effectiveness at reducing under-five mortality, two (2) showed effectiveness on mortality reduction. This, therefore, suggests that the scope and content of the current package of interventions targeted at reducing U5M will likely not achieve a rapid decline in mortality. At best, the mortality rate will be stagnant. To achieve a further decline in mortality, coverage of early initiation of breastfeeding and clean postnatal care should be increased. Further work should be done to understand the lack of association between some interventions, especially skilled delivery and U5M. Lastly, addressing issues affecting the health of children in polygamous homes and multiple births and improving National Health Insurance Scheme coverage could be beneficial.

\section{Abbreviations \\ CEM: Coarsened ExactMatching; CHPS: Community-based HealthPlanning Services; DAGs: Directed Acyclic Graphs; DPT: Diphtheria, Pertussis,and Tetanus; GDHS: Ghana Demographic andHealth Survey; GHS: Ghana Health Service; HR: Household Recode; ITN/IRS: Household InsecticideTreated Bed Nets and Indoor Residual Spraying; IPT-P: IntermittentPreventive Treatment of Malaria in Pregnancy; KR: Kids Recode; MDGs: MillenniumDevelopment Goals; MOH: Ministry of Health; NHIS: National Health InsuranceScheme; OR: Odds Ratio; SDGs: SustainableDevelopment Goals; SSA: Sub-Saharan Africa; U5M: Under-five Mortality; WHO: World HealthOrganization.}

\section{Supplementary Information}

The online version contains supplementary material available at https://doi. org/10.1186/s12887-021-02934-3.

\section{Additional file 1.}

Additional file 2.

Additional file 3.

Additional file 4

\section{Acknowledgements}

We acknowledge the International Fellowships Programme Alumni Awards for the financial support for this work. The DHS Program permitted us to use their data for the work, and we are grateful. Lastly, we are thankful to Dr. Ebenezer Owusu Adjah of Novotech, Australia, for reviewing the manuscript.

\section{Authors' contributions}

$A K, P A, B S$, and ADP conceptualized the study. AK and DD conducted the analysis. AK interpreted the results and wrote the paper. PA, BS, ADP, and DD reviewed the paper. All authors have read and approved the manuscript. 


\section{Funding}

This study was funded by the International Fellowships Programme Alumni Awards, United States of America. The funding agent had no role in the conception, design, execution, analysis, and interpretation of the study and the writing of the report.

\section{Availability of data and materials}

The datasets used and/or analyzed during the current study are available from the corresponding author on reasonable request.

\section{Declarations}

\section{Ethics issues and consent to participate}

Publically available data were used. The study did not involve human subjects as secondary data was used.

\section{Consent for publication}

Not applicable.

\section{Competing interests}

The authors declare that they have no competing interests.

\section{Author details}

${ }^{1}$ University for Development Studies, Accra, Tamale, Ghana. ${ }^{2}$ School of Public Health, University of Ghana, Legon, Accra, Ghana.

Received: 27 January 2021 Accepted: 17 September 2021 Published online: 25 October 2021

\section{References}

1. United Nations. The Millennium Development Goals Report 2015. 2015.

2. Schieber G, Cashin C, Saleh K. Health financing in Ghana: World Bank Publications; 2012.

3. Ghana Statistical Service. Ghana Demographic and Health Survey 2014. 2015.

4. Kayode GA, Grobbee DE, Koduah A, Amoakoh-Coleman M, Agyepong IA, Ansah E, et al. Temporal trends in childhood mortality in Ghana: impacts and challenges of health policies and programs. Global health action. 2016;9(1):31907.

5. Kayode GA, Ansah E, Agyepong IA, Amoakoh-Coleman M, Grobbee DE, Klipstein-Grobusch K. Individual and community determinants of neonatal mortality in Ghana: a multilevel analysis. BMC pregnancy and childbirth. 2014;14:165.

6. Akseer N, Kamali M, Bakhache N, Mirza M, Mehta S, Al-Gashm S, et al. Status and drivers of maternal, newborn, child and adolescent health in the Islamic world: a comparative analysis. The Lancet. 2018;391(10129):1493-512.

7. Quansah E, Ohene LA, Norman L, Mireku MO, Karikari TK. Social Factors Influencing Child Health in Ghana. Plos One. 2016;11(1):e0145401-e.

8. Ruducha J, Mann C, Singh NS, Gemebo TD, Tessema NS, Baschieri A, et al. How Ethiopia achieved Millennium Development Goal 4 through multisectoral interventions: a Countdown to 2015 case study. The Lancet Global Health. 2017;5(11):e1142-e51.

9. Jones G, Steketee RW, Black RE, Bhutta ZA, Morris SS, Group BCSS. How many child deaths can we prevent this year? The lancet. 2003;362(9377):65-71.

10. Black RE, Levin C, Walker N, Chou D, Liu L, Temmerman M. Reproductive, maternal, newborn, and child health: key messages from Disease Control Priorities 3rd Edition. The Lancet. 2016;388(10061):2811-24.

11. Ghana Statistical Service (GSS), Ghana Health Service (GHS), ICF I. Ghana Demographic and Health Survey 2014. Rockville, Maryland, USA: GSS, GHS, and ICF; 2015.

12. Ayalneh AA, Fetene DM, Lee TJ. Inequalities in health care utilization for common childhood illnesses in Ethiopia: evidence from the 2011 Ethiopian Demographic and Health Survey. International journal for equity in health. 2017;16(1):67.

13. Tefera W, Tesfaye H, Bekele A, Kayessa E, Waltensperger KZ, Marsh DR. Factors influencing the low utilization of curative child health services in Shebedino District, Sidama Zone, Ethiopia. Ethiopian medical journal. 2014;52(Suppl 3):109-17.

14. Young M, Wolfheim C, Marsh DR, Hammamy D. World Health Organization/United Nations Children's Fund joint statement on integrated community case management: an equity-focused strategy to improve access to essential treatment services for children. The American journal of tropical medicine and hygiene. 2012;87(5_Suppl):6-10.

15. Abir T, Agho KE, Page AN, Milton AH, Dibley MJ. Risk factors for under-5 mortality: evidence from Bangladesh Demographic and Health Survey, 2004-2011. BMJ open. 2015;5(8):e006722.

16. Sazawal S, Black RE, Ramsan M, Chwaya HM, Stoltzfus RJ, Dutta A, et al. Effects of routine prophylactic supplementation with iron and folic acid on admission to hospital and mortality in preschool children in a high malaria transmission setting: community-based, randomised, placebo-controlled trial. Lancet (London, England). 2006;367(9505):133-43.

17. Aaby P, Ravn H, Fisker AB, Rodrigues A, Benn CS. Is diphtheria-tetanuspertussis (DTP) associated with increased female mortality? A metaanalysis testing the hypotheses of sex-differential non-specific effects of DTP vaccine. Transactions of the Royal Society of Tropical Medicine and Hygiene. 2016;110(10):570-81.

18. Mogensen SW, Andersen A, Rodrigues A, Benn CS, Aaby P. The introduction of diphtheria-tetanus-pertussis and oral polio vaccine among young infants in an urban African community: a natural experiment. EBioMedicine. 2017;17:192-8.

19. Klein SL, Shann F, Moss WJ, Benn CS, Aaby P. RTS, S malaria vaccine and increased mortality in girls. mBio. 2016;7(2):1.

20. Clemens J, Moorthy V. Implementation of RTS, S/AS01 malaria vaccine - the need for further evidence. N Engl J Med. 2016;374:2597.

21. Mosley WH, Chen LC. An analytical framework for the study of child survival in developing countries. Population and development review. 1984; 10:25-45

22. Rothman KJ, Greenland S. Causation and causal inference in epidemiology. American journal of public health. 2005;95(S1):S144-S50.

23. lacus S, King G, Porro G. CEM: Coarsened exact matching software. 2009.

24. lacus SM, King G, Porro G. Matching for causal inference without balance checking. 2008

25. lacus SM, King G, Porro G. CEM: software for coarsened exact matching. 2009.

26. lacus SM, King G, Porro G. Causal inference without balance checking: Coarsened exact matching. Political analysis. 2012;20(1):1-24

27. Ghana Statistical Service. 2010 Population \& Housing Census National Analytical Report. 2013.

28. Ghana Statistical Service. Ghana Living Standards Survey. 2014 July 5, 2019.

29. Buxton CNA. Ghanaian junior high school adolescents dietary practices and food preferences: implications for public health concern. Journal of Nutrition \& Food Sciences. 2014;4(5):1.

30. Intiful F, Lartey A. Breakfast habits among school children in selected communities in the eastern region of Ghana. Ghana Medical Journal. 2014;48(2):71-7.

31. Galbete C, Nicolaou M, Meeks KA, de-Graft Aikins A, Addo J, Amoah SK, et al. Food consumption, nutrient intake, and dietary patterns in Ghanaian migrants in Europe and their compatriots in Ghana. Food \& Nutrition Research. 2017;61(1):1341809.

32. Oti J, Eshun G. Dietary Habits and Nutritional Status of Undergraduate Students of Winneba Campus of University of Education, Winneba, Ghana. Jou Food Sci\&Nutri: JFSN-109 DOI: 1046715/jfsn2020. 2020;10.

33. Dwomoh D, Amuasi S, Agyabeng K, Incoom G, Alhassan Y, Yawson AE. Understanding the determinants of infant and under-five mortality rates: a multivariate decomposition analysis of Demographic and Health Surveys in Ghana, 2003, 2008 and 2014. BMJ Global Health. 2019;4(4):e001658.

34. Ghana Statistical Service (GSS), Ghana Health Service (GHS), ICF M. Ghana Demographic and Health Survey 2009. Rockville, Maryland, USA: GSS, GHS, and ICF; 2009.

35. Brookhart MA, Schneeweiss S, Rothman KJ, Glynn RJ, Avorn J, Stürmer T. Variable selection for propensity score models. American Journal of Epidemiology. 2006;163(12):1149-56. 
36. Stuart EA. Matching methods for causal inference: A review and a look forward. Statistical science: a review journal of the Institute of Mathematical Statistics. 2010;25(1):1.

37. StataCorp L. Stata statistical software: Release 13. 2013.

38. Ghana Statistical Service. Ghana Demographic and Health Survey 2008. Report. 2009.

39. Seward N, Osrin D, Li L, Costello A, Pulkki-Brännström A-M, Houweling TA, et al. Association between clean delivery kit use, clean delivery practices, and neonatal survival: pooled analysis of data from three sites in South Asia. PLoS medicine. 2012;9(2):e1001180.

40. Messeret ES, Masresha B, Yakubu A, Daniel F, Mihigo R, Nshimirimana D, et al. Maternal and Neonatal Tetanus Elimination (MNTE) in The WHO African Region. Journal of immunological sciences. 2018(15):103.

41. Shifa GT, Ahmed AA, Yalew AW. Maternal and child characteristics and health practices affecting under-five mortality: A matched case control study in Gamo Gofa Zone, Southern Ethiopia. PLoS ONE. 2018;13(8):1-17.

42. Issaka Al, Agho KE, Renzaho AMN. The Impact of Internal Migration on under-Five Mortality in 27 Sub-Saharan African Countries. PLoS ONE. 2016;11(10):1-16.

43. Millogo O, Doamba JEO, Sié A, Utzinger J, Vounatsou P. Geographical variation in the association of child, maternal and household health interventions with under-five mortality in Burkina Faso. PLoS ONE. 2019;14(7):1-17.

44. Edmond KM, Zandoh C, Quigley MA, Amenga-Etego S, Owusu-Agyei S, Kirkwood BR. Delayed breastfeeding initiation increases risk of neonatal mortality. Pediatrics. 2006;117(3):e380-e6.

45. Raihana S, Dibley MJ, Rahman MM, Tahsina T, Siddique MAB, Rahman QS, et al. Early initiation of breastfeeding and severe illness in the early newborn period: An observational study in rural Bangladesh. PLoS Medicine. 2019;16(8):1-17.

46. Nambuusi BB, Ssempiira J, Makumbi FE, Utzinger J, Kasasa S, Vounatsou P. Geographical variations of the associations between health interventions and all-cause under-five mortality in Uganda. BMC Public Health. 2019;19(1):1330-

47. Abdullah A, Hort K, Butu Y, Simpson L. Risk factors associated with neonatal deaths: a matched case-control study in Indonesia. Global health action. 2016:9(1):30445.

48. Clemens J, Elyazeed RA, Rao M, MEngg M, Savarino S, Morsy BZ, et al. Early initiation of breastfeeding and the risk of infant diarrhea in rural Egypt. Pediatrics. 1999;104(1):e3-e.

49. Nesbitt RC, Lohela TJ, Manu A, Vesel L, Okyere E, Edmond K, et al. Quality along the continuum: a health facility assessment of intrapartum and postnatal care in Ghana. PloS one. 2013;8(11):e81089.

50. Singh K, Brodish P, Suchindran C. A regional multilevel analysis: can skilled birth attendants uniformly decrease neonatal mortality? Maternal and child health journal. 2014;18(1):242-9.
51. World Health Organization. Validation of maternal and neonatal tetanus elimination in Ghana, 2011. 2012;87(15):145 - 52.

52. Chowdhury AH. Determinants of under-five mortality in Bangladesh. 2013.

53. Page A-L, Hustache S, Luquero FJ, Djibo A, Manzo ML, Grais RF. Health care seeking behavior for diarrhea in children under 5 in rural Niger: results of a cross-sectional survey. BMC Public Health. 2011;11(1):389.

54. Blanchet NJ, Fink G, Osei-Akoto I. The effect of Ghana's National Health Insurance Scheme on health care utilisation. Ghana Med J. 2012;46(2):76-84

55. Krumkamp R, Sarpong N, Kreuels B, Ehlkes L, Loag W, Schwarz NG, et al. Health care utilization and symptom severity in Ghanaian children-a cross-sectional study. PLoS One. 2013;8(11):e80598.

56. Bosomprah S, Ragno PL, Gros C, Banskota H. Health insurance and maternal, newborn services utilisation and under-five mortality. Archives of Public Health. 2015;73(1):51.

57. Liu L, Oza S, Hogan D, Chu Y, Perin J, Zhu J, et al. Global, regional, and national causes of under-5 mortality in 2000-15: an updated systematic analysis with implications for the Sustainable Development Goals. The Lancet. 2016;388(10063):3027-35.

58. Kanmiki EW, Bawah AA, Agorinya I, Achana FS, Awoonor-Williams JK, Oduro AR, et al. Socio-economic and demographic determinants of under-five mortality in rural northern Ghana. BMC international health and human rights. 2014;14(1):24.

59. Omariba DWR, Boyle MH. Family structure and child mortality in subSaharan Africa: Cross-national effects of polygyny. Journal of Marriage and Family. 2007;69(2):528-43.

60. Arthi V, Fenske J. Polygamy and child mortality: Historical and modern evidence from Nigeria's Igbo. Review of Economics of the Household. 2018;16(1):97-141.

61. Kozuki N, Lee AC, Silveira MF, Victora CG, Adair L, Humphrey J, et al. The associations of birth intervals with small-for-gestational-age, preterm, and neonatal and infant mortality: a meta-analysis. BMC Public Health. 2013;13(3):S3.

62. Nakamura H, Ikeda N, Stickley A, Mori R, Shibuya K. Achieving MDG 4 in sub-Saharan Africa: what has contributed to the accelerated child mortality decline in Ghana? PloS one. 2011;6(3):e17774.

\section{Publisher's Note}

Springer Nature remains neutral with regard to jurisdictional claims in published maps and institutional affiliations.
Ready to submit your research? Choose BMC and benefit from:

- fast, convenient online submission

- thorough peer review by experienced researchers in your field

- rapid publication on acceptance

- support for research data, including large and complex data types

- gold Open Access which fosters wider collaboration and increased citations

- maximum visibility for your research: over 100M website views per year

At BMC, research is always in progress.

Learn more biomedcentral.com/submissions 